\title{
On Some Basic Theorems of Continuous Module Homomorphisms between Random Normed Modules
}

\author{
Guo Tiexin \\ School of Mathematics and Statistics, Central South University, Hunan Province, Changsha 410075, China \\ Correspondence should be addressed to Guo Tiexin; tiexinguo@csu.edu.cn
}

Received 24 April 2013; Accepted 22 July 2013

Academic Editor: Pei De Liu

Copyright (C) 2013 Guo Tiexin. This is an open access article distributed under the Creative Commons Attribution License, which permits unrestricted use, distribution, and reproduction in any medium, provided the original work is properly cited.

\begin{abstract}
We first prove the resonance theorem, closed graph theorem, inverse operator theorem, and open mapping theorem for module homomorphisms between random normed modules by simultaneously considering the two kinds of topologies-the $(\epsilon, \lambda)$ topology and the locally $L^{0}$-convex topology for random normed modules. Then, for the future development of the theory of module homomorphisms on complete random inner product modules, we give a proof with better readability of the known orthogonal decomposition theorem and Riesz representation theorem in complete random inner product modules under two kinds of topologies. Finally, to connect module homomorphism between random normed modules with linear operators between ordinary normed spaces, we give a proof with better readability of the known result connecting random conjugate spaces with classical conjugate spaces, namely, $L^{q}\left(S^{*}\right) \cong\left(L^{p}(S)\right)^{\prime}$, where $p$ and $q$ are a pair of Hölder conjugate numbers with $1 \leq p<+\infty, S$ a random normed module, $S^{*}$ the random conjugate space of $S, L^{p}(S)\left(L^{q}\left(S^{*}\right)\right)$ the corresponding $L^{p}$ (resp., $\left.L^{q}\right)$ space derived from $S$ (resp., $\left.S^{*}\right)$, and $\left(L^{p}(S)\right)^{\prime}$ the ordinary conjugate space of $L^{p}(S)$.
\end{abstract}

\section{Introduction}

The theory of probabilistic metric spaces initiated by K. Menger and subsequently developed by Schweizer and Sklar begins the study of randomizing the traditional space theory of functional analysis, where the randomness of "distance" or "norm" is expressed by probability distribution functions; compare [1]. The original notions of random metric spaces and random normed spaces occur in the course of the development of probabilistic metric and normed spaces, whereas the random distance between two points in a random metric space or the random norm of a vector in a random normed space is described by nonnegative random variables on a probability space; compare [1]. Probabilistic normed spaces are often endowed with the $(\epsilon, \lambda)$-topology and not locally convex in general; a serious obstacle to the deep development of probabilistic normed spaces is that the taditional theory of conjugate spaces does not universally apply to probabilistic normed spaces. Although the traditional theory of conjugate spaces does not universally apply to random normed spaces either, the additional measure-theoretic structure and the stronger geometric structure peculiar to a random normed space enable us to introduce the notion of an almost surely bounded random linear functional and establish its HahnBanach extension theorem, which leads to the idea of the theory of random conjugate spaces for random normed spaces; compare [2-4].

The further development of the theory of random conjugate spaces motivates us to present the important notions of random normed modules, random inner product modules, and random locally convex modules; compare [3-5]. Independent of Schweizer, Sklar, and Guo's work, in [6] Haydon et al. also introduced random normed modules as a tool for the study of ultrapowers of Lebesgue-Bochner function spaces. All the work before 2009 was carried out under the $(\epsilon, \lambda)$-topology.

In 2009, motivated by financial applications, in [7] Filipović et al. independently presented random normed modules and first applied them to the study of conditional risk measures. In particular, they introduced another kind of topology, namely, the locally $L^{0}$-convex topology, for random normed modules and random locally convex modules, and began the study of random convex analysis.

Relations between some basic results derived from the $(\epsilon, \lambda)$-topology and the locally $L^{0}$-convex topology for 
a random locally convex module were further studied in [8]. Following [8], the advantage and disadvantage of the two kinds of topologies are gradually realized and the advantage of one can complement the disadvantage of the other, which also leads to a series of recent advances $[9,10]$ and in particular to a complete random convex analysis with applications to conditional risk measures [11, 12].

Up to now, the results obtained in random metric theory are of space-theoretical nature, whereas the study of module homomorphisms between random normed modules has not been fully carried out. With the development of random metric theory, we unavoidably need a deep theory of module homomorphisms; this paper gives some basic theorems of continuous module homomorphisms. These basic theorems are known under the $(\epsilon, \lambda)$-topology, but their proofs were given before the definitive notions of random normed and inner product modules were presented in [3] so that these proofs do not have a good readability; in this paper we give better proofs and further give the versions of these basic theorems under the locally $L^{0}$-convex topology.

The remainder of this paper is organized as follows. In Section 2, we introduce some basic notions together with some simple facts subsequently used in this paper. In Section 3, we prove the resonance theorem, closed graph theorem, inverse operator theorem, and open mapping theorem for module homomorphisms between random normed modules endowed with the two kinds of topologies. In Section 4, we give a better proof of the known orthogonal decomposition theorem and Riesz representation theorem in complete random inner product modules under the two kinds of topologies for the future development of module homomorphisms between complete random inner product modules. Finally, Section 5 is devoted to a better proof of the known result connecting random conjugate spaces and ordinary conjugate spaces, namely, $\left(L^{p}(S)\right)^{\prime} \cong L^{q}\left(S^{*}\right)$.

\section{Preliminaries}

Throughout this paper, $K$ denotes the scalar field, namely, the field $R$ of real numbers or the field $C$ of complex numbers, $(\Omega, \mathscr{F}, \mu)$ a $\sigma$-finite measure space, $L^{0}(\mathscr{F}, K)$ the algebra of equivalence classes of $\mathscr{F}$-measurable $K$-valued functions on $(\Omega, \mathscr{F}, \mu), \bar{L}^{0}(\mathscr{F})$ the set of equivalence classes of extended real-valued $\mathscr{F}$-measurable functions on $(\Omega, \mathscr{F}, \mu)$ and $L^{0}(\mathscr{F}):=L^{0}(\mathscr{F}, R)$.

$\bar{L}^{0}(\mathscr{F})$ is partially ordered by $\xi \leq \eta$ if and only if $\xi^{0}(\omega) \leq$ $\eta^{0}(\omega)$ a.s., where $\xi^{0}$ and $\eta^{0}$ are arbitrarily chosen representatives of $\xi$ and $\eta$ in $\bar{L}^{0}(\mathscr{F})$, respectively. It is well known from [13] that every subset $H$ in $\bar{L}^{0}(\mathscr{F})$ has a supremum and infimum, denoted by $\vee H$ and $\wedge H$, respectively, and there are countable subsets $\left\{a_{n}, n \in N\right\}$ and $\left\{b_{n}, n \in N\right\}$ of $H$ such that $\vee H=\vee_{n \geq 1} a_{n}$ and $\wedge H=\wedge_{n \geq 1} b_{n}$. Furthermore, if, in addition, $H$ is upward directed or downward directed, then $\left\{a_{n}, n \in N\right\}$ and $\left\{b_{n}, n \in N\right\}$ can be chosen as nondecreasing and nonincreasing, respectively. In particular, $\left(L^{0}(\mathscr{F}), \leq\right)$ is conditionally complete, namely, every subset with an upper bound has a supremum.
Following are the notation and terminology frequently used in this paper:

$$
\begin{aligned}
& \bar{L}_{+}^{0}(\mathscr{F})=\left\{\xi \in \bar{L}^{0}(\mathscr{F}) \mid \xi \geq 0\right\}, \\
& L_{+}^{0}(\mathscr{F})=\left\{\xi \in L^{0}(\mathscr{F}) \mid \xi \geq 0\right\}, \\
& \bar{L}_{++}^{0}(\mathscr{F})=\left\{\xi \in L^{0}(\mathscr{F}) \mid \xi>0 \text { on } \Omega\right\},
\end{aligned}
$$

where " $\xi>0$ on $\Omega$ " means that $\xi^{0}(\omega)>0$ a.s. for an arbitrarily chosen representative $\xi^{0}$ of $\xi$.

Definition 1 (see [3]). An ordered pair $(S,\|\cdot\|)$ is called a random normed space (briefly, an RN space) over $K$ with base $(\Omega, \mathscr{F}, \mu)$ if $S$ is a linear space over $K$ and $\|\cdot\|$ is a mapping from $S$ to $L_{+}^{0}(\mathscr{F})$ such that the following three conditions are satisfied:

(RN-1) $\|x\|=0$ implies $x=\theta$ (the null in $S$ ),

(RN-2) $\|\alpha x\|=|\alpha|\|x\|$, for all $\alpha \in K$ and $x \in S$,

(RN-3) $\|x+y\| \leq\|x\|+\|y\|$, for all $x, y \in S$,

where $\|x\|$ is called the random norm of $x$. If $\|\cdot\|$ only satisfies $(\mathrm{RN}-2)$ and (RN-3), then it is called a random seminorm.

Furthermore, if, in addition, $S$ is a left module over the algebra $L^{0}(\mathscr{F}, K)$ (briefly, an $L^{0}(\mathscr{F}, K)$-module) and the following additional condition is also satisfied:

(RNM-1) $\|\xi x\|=|\xi|\|x\|$, for all $\xi \in L^{0}(\mathscr{F}, K)$ and $x \in$ $S$,

then $(S,\|\cdot\|)$ is called a random normed module (briefly, an RN module) over $K$ with base $(\Omega, \mathscr{F}, \mu)$, at which time $\|\cdot\|$ is called an $L^{0}$ norm on $S$. Similarly, if $\|\cdot\|$ only satisfies (RN-3) and (RNM-1), then it is called an $L^{0}$-seminorm on $S$.

Remark 2. In [1], the original definition of an RN space was given by only requiring $(\Omega, \mathscr{F}, \mu)$ to be a probability space and defining $\|x\|$ to be a nonnegative random variable; the corresponding $(\mathrm{RN}-1)$ to $(\mathrm{RN}-3)$ are given in the following way:

$(\mathrm{RN}-1)^{\prime}\|x\|(\omega)=0$ a.s. implies $x=\theta$,

$(\mathrm{RN}-2)^{\prime}\|\alpha x\|(\omega)=|\alpha|\|x\|(\omega)$ a.s., for all $\alpha \in K$ and $x \in S$,

$(\mathrm{RN}-3)^{\prime}\|x+y\|(\omega) \leq\|x\|(\omega)+\|y\|(\omega)$ a.s., for all $x, y \in S$.

This definition is natural and intuitive from probability theory, but $(\mathrm{RN}-1)^{\prime}$ is difficult to satisfy when we construct examples. Thus we essentially have employed Definition 1 since our work [2] by saying that measurable functions or random variables that are equal a.s. are identified; in particular since 1999 we strictly distinguish between measurable functions and their equivalence classes in writings; compare [3].

Remark 3. At outset we consider both the real and complex cases in the study of RN spaces, whereas they only consider the real case in $[6,7]$ because of their special interests; an $\mathrm{RN}$ 
module over $R$ is termed as a randomly normed $L^{0}$-module in [6] and an $L^{0}$-normed module in [7]. We still would like to continue to employ the terminology "an RN module over $K$ with base $(\Omega, \mathscr{F}, \mu)$ " in order to keep concordance with the earliest terminology used in [1].

Definition 4 (see $[3,5,14])$. Let $(S,\|\cdot\|)$ and $\left(S_{1},\|\cdot\|_{1}\right)$ be two RN spaces over $K$ with base $(\Omega, \mathscr{F}, \mu)$. A linear operator $T$ from $S$ to $S_{1}$ is said to be a.s. bounded if there exists $\xi \in L_{+}^{0}(\mathscr{F})$ such that $\|T x\|_{1} \leq \xi\|x\|$, for all $x \in S$, in which case $\|T\|$ is defined to be $\wedge\left\{\xi \in L_{+}^{0}(\mathscr{F}) \mid\|T x\|_{1} \leq \xi\|x\|, \forall x \in S\right\}$, called the random norm of $T$. Denote the linear space of a.s. bounded linear operators from $S$ to $S_{1}$ by $B\left(S, S_{1}\right)$; then $\left(B\left(S, S_{1}\right),\|\cdot\|\right)$ still becomes an RN space over $K$ with base $(\Omega, \mathscr{F}, \mu)$ when $\|T\|$ is defined as above for every $T \in B\left(S, S_{1}\right)$. In particular, when $S_{1}=L^{0}(\mathscr{F}, K)$ and $\|\cdot\|_{1}=|\cdot|$ (namely, the absolute value mapping), $S^{*}:=B\left(S, S_{1}\right)$ is called the random conjugate space of $S$ and an element in $S^{*}$ is called an a.s. bounded random linear functional on $S$.

Remark 5. When $\left(S_{1},\|\cdot\|_{1}\right)$ in Definition 4 is an $\mathrm{RN}$ module, $B\left(S, S_{1}\right)$ automatically becomes an $\mathrm{RN}$ module under the module operation $(\xi \cdot T)(x)=\xi \cdot(T(x))$, for all $\xi \epsilon$ $L^{0}(\mathscr{F}, K), T \in B\left(S, S_{1}\right)$, and $x \in S$. When $S$ and $S_{1}$ are both RN modules, in [6] $B\left(S, S_{1}\right)$ is used to stand for the $L^{0}(\mathscr{F}, K)$ module of a.s. bounded module homomorphisms from $S$ to $S_{1}$; we will show that in the special case an $a$.s. bounded linear operator must be a module homomorphism. Therefore, the two implications of $B\left(S, S_{1}\right)$ coincide in this case.

As in the classical functional analysis, we can similarly define a conjugate operator $T^{*}: S_{2}^{*} \rightarrow S_{1}^{*}$ for an a.s. bounded linear operator $T$ from $\left(S_{1},\|\cdot\|_{1}\right)$ to $\left(S_{2},\|\cdot\|_{2}\right)$ as follows: $\left(T^{*} f\right)(x)=f(T x)$, for all $f \in S_{2}^{*}, x \in S_{1}$. From the Hahn-Banach theorem for a.s. bounded random linear functional established in [2] (also see [8]), one has that $\left\|T^{*}\right\|=$ $\|T\|$.

For the sake of convenience, let us recall some notation and terminology in the theory of probabilistic normed spaces.

Definition 6 (see [1]). A function $T:[0,1] \times[0,1] \rightarrow[0,1]$ is called a weak $t$-norm if the following are satisfied:

$(\mathrm{t}-1) T(a, b)=T(b, a)$, for all $a, b \in[0,1]$,

(t-2) $T(a, b) \leq T(c, d)$, for all $a, b, c, d \in[0,1]$ with $a \leq c, b \leq d$,

$(\mathrm{t}-3) T(1,0)=0, T(1,1)=1$.

A weak $t$-norm $T:[0,1] \times[0,1] \rightarrow[0,1]$ is called a $t$-norm if the following two additional conditions are satisfied:

(t-4) $T(a, T(b, c))=T(T(a, b), c)$, for all $a, b, c \in$ $[0,1]$,

$(\mathrm{t}-5) T(1, a)=a$, for all $a \in[0,1]$.
Although $t$-norms are widely used in the theory of probabilistic metric spaces, weak $t$-norms have their own advantages, for example, for a family $\left\{T_{\alpha}, \alpha \in \wedge\right\}$ of weak $t$ norms, $T$ defined by $T(a, b)=\sup \left\{T_{\alpha}(a, b): \alpha \in \wedge\right\}$, for all $a, b \in[0,1]$, is still a weak $t$-norm, whereas this is not true for $t$-norms.

Throughout this paper, $\Delta=\{F:[-\infty,+\infty] \rightarrow[0,1] \mid$ $F(-\infty)=0, F(+\infty)=1, F$ is nondecreasing and left continuous on $(-\infty,+\infty)\}, D=\left\{F \in \Delta \mid \lim _{x \rightarrow-\infty} F(x)=0\right.$ and $\left.\lim _{x \rightarrow+\infty} F(x)=1\right\}, \Delta^{+}=\{F \in \Delta \mid F(0)=0\}$, and $D^{+}=$ $\{F \in D \mid F(0)=0\}$. For extended real random variable $\xi$ on a probability space $(\Omega, \mathscr{F}, P)$, its (left continuous) distribution function $N_{\xi}$ is defined by $N_{\xi}(t)=P\{\omega \in \Omega \mid \xi(\omega)<t\}$, for all $t \in[-\infty,+\infty)$ and $N_{\xi}(+\infty)=1$.

In particular, $\epsilon_{0}$ stands for the distribution function defined by $\epsilon_{0}(t)=1$ when $t>0$ and $\epsilon_{0}(t)=0$ when $t \leq 0$; namely, $\epsilon_{0}$ is the distribution function of the constant 0 .

Definition 7 (see [1]). A triple $(S, \mathcal{N}, T)$ is called a Menger probabilistic normed space (briefly, an M-PN space) over $K$ if $S$ is a linear space over $K, \mathcal{N}$ is a mapping from $S$ to $D^{+}$, and $T$ is a weak $t$-norm such that the following are satisfied:

$(\mathrm{MPN}-1) N_{x}=\epsilon_{0}$ if and only if $x=\theta$ (the null element in $S$ ),

$(\mathrm{MPN}-2) N_{\alpha x}(t)=N_{x}(t /|\alpha|)$, for all $t \geq 0, \quad \alpha \in K \backslash$ $\{0\}$ and $x \in S$,

(MPN-3) $N_{x+y}(u+v) \geq T\left(N_{x}(u), N_{y}(v)\right)$, for all $u, v \geq$ 0 and $x, y \in S$.

Here $N_{x}:=\mathscr{N}(x)$ is called the probabilistic norm of $x$.

For an M-PN space $(S, \mathcal{N}, T)$, let $\mathscr{T}=\{\widetilde{T} \mid \widetilde{T}$ is a weak $t$ norm such that $(S, \mathcal{N}, \widetilde{T})$ is an M-PN space $\}$, and define $\widehat{T}$ : $[0,1] \times[0,1] \rightarrow[0,1]$ by $\widehat{T}(a, b)=\sup \{\widetilde{T}(a, b) \mid \widetilde{T} \in \mathscr{T}\}$, for all $a, b \in[0,1]$; then it is very easy to see that $\widehat{T} \in \mathscr{T}$. $\widehat{T}$ is called the largest weak $t$-norm of $(S, \mathcal{N})$ such that $(S, \mathcal{N})$ is an M-PN space under $\widehat{T}$. From now on, for an M-PN space $(S, \mathcal{N}, T)$, we always assume that $T$ is the largest weak $t$-norm of $(S, \mathcal{N})$.

In [15], LaSalle introduced the notion of a pseudonormed linear space: let $S$ be a linear space over $K$ and $\left\{p_{\alpha}\right\}_{\alpha \in \Lambda}$ a family of mappings from $S$ to $R^{+}:=[0,+\infty)$ and indexed by a directed set $\wedge$; then $\left(S,\left\{p_{\alpha}\right\}_{\alpha \in \Lambda}\right)$ is called a pseudonormed linear space if the following are satisfied:

(PNS-1) $p_{\alpha}(\beta x)=|\beta| p_{\alpha}(x)$, for all $\beta \in K, x \in S$, and $\alpha \in \wedge$,

(PNS-2) $p_{\alpha_{1}}(x) \leq p_{\alpha_{2}}(x)$, for all $\alpha_{1}, \alpha_{2} \in \wedge$ such that $\alpha_{1} \leq \alpha_{2}, x \in S$,

(PNS-3) for any $\alpha \in \wedge$, there exists $\alpha^{\prime} \in \wedge$ such that $p_{\alpha}(x+y) \leq p_{\alpha^{\prime}}(x)+p_{\alpha^{\prime}}(y)$, for all $x, y \in S$.

Let $\left(S,\left\{p_{\alpha}\right\}_{\alpha \in \Lambda}\right)$ be a pseudonormed linear space. For any $\epsilon>0$ and $\alpha \in \wedge$, let $U_{\theta}(\alpha, \epsilon)=\left\{x \in S \mid p_{\alpha}(x)<\epsilon\right\}$. Then $\mathscr{U}_{\theta}=\left\{U_{\theta}(\alpha, \epsilon) \mid \alpha \in \wedge, \epsilon>0\right\}$ is a local base at the null element $\theta$ of some linear topology for $S$, called the linear topology induced by $\left\{p_{\alpha}\right\}_{\alpha \in \wedge}$. Conversely any linear topology 
for $S$ can be induced by some $\left\{p_{\alpha}\right\}_{\alpha \in \Lambda}$ such that $\left(S,\left\{p_{\alpha}\right\}_{\alpha \in \Lambda}\right)$ is a pseudonormed linear space.

To connect an M-PN space $(S, \mathcal{N}, T)$ to a pseudonormed linear space, for each $r \in(0,1)$, define $p_{r}: S \rightarrow[0,+\infty)$ by $p_{r}(x)=\sup \left\{t \geq 0 \mid N_{x}(t)<r\right\}$, for all $x \in S$. Then we have the following.

Theorem 8 (see $[16])$. Let $(S, \mathcal{N}, T)$ be an $M-P N$ space. Then one has the following statements.

(1) $\sup _{0<a<1} T(a, a)=1$ if and only if $\left(S,\left\{p_{r}\right\}_{r \in(0,1)}\right)$ is a pseudonormed linear space; namely, for each $r \in(0,1)$ there exists $s \in(0,1)$ such that $p_{r}(x+y) \leq p_{s}(x)+$ $p_{s}(y)$, for all $x, y \in S$.

(2) $T \geq$ Min, namely, $T(a, b) \geq \min (a, b)$, for all $a, b \in$ $[0,1]$, if and only if $p_{r}$ is a seminorm on $S$ for each $r \in$ $(0,1)$; namely, $\left(S,\left\{p_{r}\right\}_{r \in(0,1)}\right)$ is a $B_{0}$-type space.

(3) $T(a, b)=1$ for all $a, b \in[0,1]$ such that $a \cdot b>0$ if and only if there exists a norm $\|\cdot\|$ on $S$ such that $p_{r}=\|\cdot\|$, for all $r \in(0,1)$.

Theorem 8 was first studied in [17] in terms of isometric metrization and first given and strictly proved in its present form in [16].

Proposition 9 (see [1]). Let $(S, \mathcal{N}, T)$ be an $M-P N$ space such that $\sup _{0<a<1} T(a, a)=1$. For any positive numbers $\epsilon$ and $\lambda$ with $0<\lambda<1$, let $U_{\theta}(\epsilon, \lambda)=\left\{x \in S \mid N_{x}(\epsilon)>1-\lambda\right\}$; then $\mathcal{U}_{\theta}=\left\{U_{\theta}(\epsilon, \lambda) \mid \epsilon>0,0<\lambda<1\right\}$ forms a local base at $\theta$ of some metrizable linear topology for $S$, called the $(\epsilon, \lambda)$-topology induced by $\mathcal{N}$.

From Theorem 8, one can easily see that the $(\epsilon, \lambda)$ topology for an M-PN space $(S, \mathcal{N}, T)$ with $\sup _{0<a<1} T(a, a)=$ 1 is exactly the one induced by the family $\left\{p_{r}\right\}_{r \in(0,1)}$ of pseudonorms. Therefore as far as the study of linear homeomorphic invariants for a metrizable linear topological space is concerned, the theory of an M-PN space $(S, \mathcal{N}, T)$ with $\sup _{0<a<1} T(a, a)=1$ and the theory of pseudonormed linear space $\left(S,\left\{p_{r}\right\}_{r \in(0,1)}\right)$ are equivalent to each other, and hence either of them is also equivalent to the theory of a quasinormed space (see [18] for a quasinormed space) since a metrizable linear topology can be equivalently induced by a quasinorm as well as a family of pseudonorms such as $\left\{p_{r}\right\}_{r \in(0,1)}$. We find that the three kinds of frameworks have their own advantages and all will be used in this paper.

Definition 10 (see [1]). Let $(S, \mathcal{N}, T)$ be an M-PN space with $\sup _{0<a<1} T(a, a)=1$ and $A$ a subset of $S . D_{A}:[-\infty,+\infty] \rightarrow$ $[0,1]$ is defined by $D_{A}(t)=\sup _{r<t}\left(\inf \left\{N_{x}(r): x \in A\right\}\right)$, for all $t \in(-\infty,+\infty), D_{A}(-\infty)=0$, and $D_{A}(+\infty)=1$, called the probabilistic diameter of $A$. If $D_{A} \in D^{+}$, then $A$ is said to be probabilistically bounded.

Proposition 11 below is a straightforward verification by definition.

Proposition 11. Let $(S, \mathcal{N}, T)$ and $A$ be the same as in Definition 10. Then $A$ is probabilistically bounded if and only if $A$ is bounded with respect to the $(\epsilon, \lambda)$-topology (namely, $A$ can be absorbed by every $(\epsilon, \lambda)$-neighborhood of the null $\theta$ ).

Let $(\Omega, \mathscr{F}, \mu)$ be a probability space and $(S,\|\cdot\|)$ an RN space over $K$ with base $(\Omega, \mathscr{F}, \mu)$. Define $\mathcal{N}: S \rightarrow D^{+}$by $N_{x}(t)=\mu\{\omega \in \Omega \mid\|x\|(\omega)<t\}$, for all $t \geq 0$ and $x \in S$; namely, $N_{x}$ is the distribution of $\|x\|$; then $(S, \mathcal{N}, T)$ is an M-PN space with $T \geq W$, where $W(a, b)=\max (a+b-$ $1,0)$, for all $a, b \in[0,1] .(S, \mathcal{N}, T)$ is called the M-PN space determined by $(S,\|\cdot\|)$; the $(\epsilon, \lambda)$-topology for $(S, \mathcal{N}, T)$ is also called the $(\epsilon, \lambda)$-topology for $(S,\|\cdot\|)$.

When $(\Omega, \mathscr{F}, \mu)$ is a $\sigma$-finite measure space, let $\mathscr{F}_{+}=\{A \epsilon$ $\mathscr{F} \mid 0<\mu(A)<+\infty\}$; then the following definition is a slight generalization of the case when $(\Omega, \mathscr{F}, \mu)$ is a probability space.

Definition 12 (see [3]). Let $(S,\|\cdot\|)$ be an $\mathrm{RN}$ space over $K$ with base $(\Omega, \mathscr{F}, \mu)$. For $A \in \mathscr{F}_{+}, \epsilon>0$ and $\lambda>0$, let $U_{\theta}(A, \epsilon, \lambda)=$ $\{x \in S \mid \mu\{\omega \in A \mid\|x\|(\omega)<\epsilon\}>\mu(A)-\lambda\}$. Then $\mathscr{U}_{\theta}=$ $\left\{U_{\theta}(A, \epsilon, \lambda) A \in \mathscr{F}_{+}(A), \epsilon>0, \lambda>0\right\}$ forms a local base at $\theta$ of some metrizable linear topology for $S$, called the $(\epsilon, \lambda)$ topology for $S$ induced by $\|\cdot\|$.

Proposition 13 below is a straightforward verification by definition.

Proposition 13. Let $(S,\|\cdot\|)$ be an $R N$ space over $K$ with base $(\Omega, \mathscr{F}, \mu)$ and $\left\{A_{n} \mid n \in N\right\}$ a countable partition of $\Omega$ to $\mathscr{F}$ such that $0<\mu\left(A_{n}\right)<+\infty$. Then one has the following.

(1) A sequence $\left\{x_{n} x \in N\right\}$ in $S$ converges in the $(\epsilon, \lambda)$ topology to $x$ in $S$ if and only if $\left\{\left\|x_{n}-x\right\| \mid n \in N\right\}$ converges to 0 locally in measure; namely, $\left\{\left\|x_{n}-x\right\| \mid\right.$ $n \in N\}$ converges to 0 in measure $\mu$ on each $A \in \mathscr{F}_{+}$.

(2) The $(\epsilon, \lambda)$-topology for $S$ is exactly the linear topology induced by the quasinorm $|\|\cdot\||$ defined by $|\|x\||=$ $\sum_{n=1}^{\infty}\left(1 / 2^{n}\right) \int_{A_{n}}(\|x\|) /(1+\|x\|) d \mu$ for all $x \in S$.

(3) Let $P: \mathscr{F} \rightarrow[0,1]$ be defined by $P(A)=$ $\sum_{n=1}^{\infty}\left(1 / 2^{n}\right)\left(\mu\left(A \cap A_{n}\right) / \mu\left(A_{n}\right)\right)$; then $P$ is a probability measure equivalent to $\mu$ and $(S,\|\cdot\|)$ has the same $(\epsilon, \lambda)$ topology whether $(S,\|\cdot\|)$ is regarded as an $R N$ space with base $(\Omega, \mathscr{F}, \mu)$ or $(\Omega, \mathscr{F}, P)$.

Remark 14 . When $(\Omega, \mathscr{F}, \mu)$ is a $\sigma$-finite measure space, the $(\epsilon, \lambda)$-topology for the special $\mathrm{RN}$ space $\left(L^{0}(\mathscr{F}),|\cdot|\right)$ is exactly the topology of convergence locally in measure. But the topology of convergence in measure is not a linear topology in general, so we choose the $(\epsilon, \lambda)$-topology since not only is it a linear topology but also its convergence has almost all the nice properties of convergence in measure (see (1) of Proposition 13). (3) of Proposition 13 shows that we can always assume the base space of an RN space to be a probability space when only the linear homeomorphic invariants or those independent of the special choice of $\mu$ and $P$ are studied. Finally, independently of B Schweizer and Sklar's work [1], the $(\epsilon, \lambda)$-topology is also introduced in [6], called the $L^{0}$-topology. 
Definition 15 (see $[3,5,14])$. Let $(S,\|\cdot\|)$ be an RN space over $K$ with base $(\Omega, \mathscr{F}, \mu)$ and $A$ a subset of $S$. $A$ is said to be a.s. bounded if $\vee\{\|a\|: a \in A\} \in L_{+}^{0}(\mathscr{F})$.

In the sequel, the $(\epsilon, \lambda)$-topology for every $\mathrm{RN}$ space is always denoted by $\mathscr{T}_{\epsilon, \lambda}$ and the quasinorm for every $\mathrm{RN}$ space is always denoted by $|\|\cdot\||$ defined as in (2) of Proposition 13 when no confusion occurs.

Proposition 16 (see [3]). Let $(S,\|\cdot\|)$ be an $R N$ space with base $(\Omega, \mathscr{F}, \mu)$ and $A$ a subset of $S$ such that $\{\|a\|: a \in$ $A\}$ is upward directed. Then $A$ is a.s. bounded if and only if it is $\mathscr{T}_{\epsilon, \lambda}$-bounded, at which time and when $(\Omega, \mathscr{F}, \mu)$ is a probability space, $D_{A}=N_{\xi}$, where $\xi=\vee\{\|a\|: a \in A\}$ and $N_{\xi}$ is the distribution function of $\xi$.

Proof. We can, without loss of generality, assume that $(\Omega, \mathscr{F}, \mu)$ is a probability space. Necessity is clear. We prove the sufficiency as follows.

Since there exists a sequence $\left\{x_{n}, n \in N\right\}$ in $A$ such that $\left\{\left\|x_{n}\right\|, n \in N\right\}$ converges a.s. to $\xi$ in a nondecreasing manner. Let $(S, \mathcal{N}, T)$ be the M-PN space determined by $(S,\|\cdot\|)$; then $\left\{N_{x_{n}}, n \in N\right\}$ converges weakly to $N_{\xi}$; it is easy to check that $N_{\xi}=D_{\left\{x_{n}, n \in N\right\}}$ (namely, the probabilistic diameter of $\left\{x_{n}, n \in\right.$ $N\}$ ), and hence $N_{\xi} \geq D_{A}$. But $N_{\xi} \leq D_{A}$ is clear, then $N_{\xi}=$ $D_{A}$. Since $A$ is $\mathscr{T}_{\xi, \lambda}$-bounded, $D_{A} \in D^{+}$, which shows that $\xi \in L_{+}^{0}(\mathscr{F})$.

Proposition 17 below gives a very general condition for $\{\|a\|: a \in A\}$ to be upward directed or downward directed.

Proposition 17. Let $(S,\|\cdot\|)$ be an $R N$ module with base $(\Omega, \mathscr{F}, \mu)$ and $A$ a subset of $S$ such that $\widetilde{I}_{D} A+\widetilde{I}_{D^{C}} A \subset A$ for any $D \in \mathscr{F}$, where $D^{C}=\Omega \backslash D$ and $\widetilde{I}_{D}$ stands for the equivalence class of the characteristic function of $D$. Then $\{\|a\|: a \in A\}$ is both upward and downward directed.

Proof. We only prove that $\{\|a\|: a \in A\}$ is upward directed; the case of being downward directed is similar. For any $a_{1}, a_{2} \in A$, let $D=\left\{\omega \in \Omega \mid\left\|a_{1}\right\|^{0}(\omega) \leq\left\|a_{2}\right\|^{0}(\omega)\right\}$, where $\left\|a_{1}\right\|^{0}$ and $\left\|a_{2}\right\|^{0}$ are arbitrarily chosen representatives of $\left\|a_{1}\right\|$ and $\left\|a_{2}\right\|$, respectively. Then $a_{3}:=\widetilde{I}_{D} a_{2}+\widetilde{I}_{D^{C}} a_{1}$ is such that $\left\|a_{3}\right\|=\widetilde{I}_{D}\left\|a_{2}\right\|+\widetilde{I}_{D^{C}}\left\|a_{1}\right\|=\left\|a_{1}\right\| \vee\left\|a_{2}\right\|$. Since $a_{3} \in A$, the proof is complete.

It is easy to see that $\left(L^{0}(\mathscr{F}, K), \mathscr{T}_{\epsilon, \lambda}\right)$ is a topological algebra over $K$ and $\left(S, \mathscr{T}_{\epsilon, \lambda}\right)$ is a topological module over $\left(L^{0}(\mathscr{F}, K), \mathscr{T}_{\epsilon, \lambda}\right)$ when $(S,\|\cdot\|)$ is an RN module over $K$ with base $(\Omega, \mathscr{F}, \mu)$. In 2009, another kind of topology for an $\mathrm{RN}$ module was introduced in [7].

Definition 18 (see [7]). Let $(S,\|\cdot\|)$ be an RN module over $K$ with base $(\Omega, \mathscr{F}, \mu)$. A subset $G$ of $S$ is called a $\mathscr{T}_{c}$-open set if for each $x \in G$ there exists some $\epsilon \in L_{++}^{0}(\mathscr{F})$ such that $x+U_{\theta}(\epsilon) \subset G$, where $U_{\theta}=\{y \in S \mid\|y\| \leq \epsilon\}$. Denote by $\mathscr{T}_{c}$ the family of $\mathscr{T}_{c}$-open sets; then $\mathscr{T}_{c}$ forms a Hausdorff topology for $S$, called the locally $L^{0}$-convex topology induced by $\|\cdot\|$.
It is easy to check that the locally $L^{0}$-convex topology is much stronger than the $(\epsilon, \lambda)$-topology for a given $\mathrm{RN}$ module; $\left(L^{0}(\mathscr{F}, K), \mathscr{T}_{c}\right)$ is, however, only a topological ring since it is unnecessarily a linear topological space (see [7]). Furthermore, for an RN module $(S,\|\cdot\|)$ over $K$ with base $(\Omega, \mathscr{F}, \mu),\left(S, \mathscr{T}_{c}\right)$ is a topological module over the topological ring $\left(L^{0}(\mathscr{F}, K), \mathscr{T}_{c}\right)$; compare [7]. From now on, the locally $L^{0}$-convex topology for every RN module is always denoted by $\mathscr{T}_{c}$ when no confusion occurs.

Definition 19. Let $S$ be an $L^{0}(\mathscr{F}, K)$-module. A subset $G$ of $S$ is said to be $L^{0}$-convex if $\xi x+(1-\xi) y \in G$, for all $x, y \in G$ and $\xi \in L_{+}^{0}(\mathscr{F})$ with $0 \leq \xi \leq 1$. A subset $G$ of $S$ is said to be $L^{0}$-balanced if $\xi x \in G$ for all $x \in G$ and $\xi \in L^{0}(\mathscr{F}, K)$ with $|\xi| \leq 1$. A subset $G$ of $S$ is said to be $L^{0}$-absorbed by a subset $H$ of $S$ if there exists some $\xi \in L_{++}^{0}(\mathscr{F})$ such that $\eta G \subset H$ for all $\eta \in L^{0}(\mathscr{F}, K)$ with $|\eta| \leq \xi$. Furthermore, if a subset $G$ of $S L^{0}$ absorbs every element of $S$, then $G$ is said to be $L^{0}$-absorbent.

Definition 20 (see [12]). Let $(S,\|\cdot\|)$ be an RN module and $A$ a subset of $S$. $A$ is said to be $\mathscr{T}_{c}$-bounded if $A$ is $L^{0}$-absorbed by every $\mathscr{T}_{c}$-neighborhood of the null element.

It is also very easy to see that a subset of an $\mathrm{RN}$ module is $\mathscr{T}_{c}$-bounded if and only if it is a.s. bounded.

For the sake of convenience, $I_{A}$ always denotes the characteristic function of $A \in \mathscr{F}$ and $\widetilde{I}_{A}$ the equivalence class of $I_{A}$. As usual, $\{B \in \mathscr{F} \mid \mu(A \Delta B)=0\}$ is called the equivalence class of $A \in \mathscr{F}$, denoted by $\widetilde{A}$; we sometimes also use $I_{\widetilde{A}}$ for $\widetilde{I}_{A}$.

Theorem 21 below is a formal generalization of the corresponding results given in $[5,19]$ for a random linear functional; it was already frequently employed in $[12,14]$ but does not have yet a better proof; here we give a better proof. From now on, for convenience we always denote by $(S, N)$ the $\mathrm{M}-\mathrm{PN}$ space determined by a given $\mathrm{RN}$ space $(S,\|\cdot\|)$.

Theorem 21. Let $\left(S_{1},\|\cdot\|_{1}\right)$ and $\left(S_{2},\|\cdot\|_{2}\right)$ be two RN modules over $K$ with base $(\Omega, \mathscr{F}, \mu)$ and $T: S_{1} \rightarrow S_{2}$ a linear operator. Then one has the following:

(1) $T$ is a.s. bounded if and only if $T$ is a continuous module homomorphism from $\left(S_{1}, \mathscr{T}_{\epsilon, \lambda}\right)$ to $\left(S_{2}, \mathscr{T}_{\epsilon, \lambda}\right)$;

(2) $T$ is a.s. bounded if and only if $T$ is a continuous module homomorphism from $\left(S_{1}, \mathscr{T}_{c}\right)$ to $\left(S_{2}, \mathscr{T}_{c}\right)$.

Proof.

(1) Necessity. Since $T$ is a.s. bounded, $T$ must be continuous from $\left(S_{1}, \mathscr{T}_{\epsilon, \lambda}\right)$ to $\left(S_{2}, \mathscr{T}_{\epsilon, \lambda}\right)$; it remains to prove that $T$ is also a module homomorphism; it suffices to prove that $T\left(\widetilde{I}_{A} x\right)=$ $\widetilde{I}_{A} T(x)$, for all $x \in S_{1}$ and $A \in \mathscr{F}$, since $T$ is linear. Since $\left\|T\left(\widetilde{I}_{A} x\right)\right\|_{2} \leq\|T\| \cdot\left\|\widetilde{I}_{A} x\right\|_{1}=\widetilde{I}_{A}\|T\| \cdot\|x\|_{1}, \widetilde{I}_{A^{c}} \cdot T\left(\widetilde{I}_{A} x\right)=\theta$, for all $A \in \mathscr{F}$. Then $T\left(\widetilde{I}_{A} x\right)=\left(\widetilde{I}_{A}+\widetilde{I}_{A^{c}}\right) \cdot T\left(\widetilde{I}_{A} x\right)=\widetilde{I}_{A} \cdot T\left(\widetilde{I}_{A} x\right)$, for all $A \in \mathscr{F}$. On the other hand, $\widetilde{I}_{A} T(x)=\widetilde{I}_{A} \cdot T\left(\widetilde{I}_{A} x+\right.$ $\left.\widetilde{I}_{A^{c}} x\right)=\widetilde{I}_{A} \cdot T\left(\widetilde{I}_{A} x\right)$, for all $x \in S_{1}$ and for all $A \in \mathscr{F}$. So, $\widetilde{I}_{A} \cdot T(x)=T\left(\widetilde{I}_{A} x\right)$. 
Sufficiency. $S_{1}(1):=\left\{x \in S_{1} \mid\|x\|_{1} \leq 1\right\}$ is a.s. bounded, and hence also $\mathscr{T}_{\epsilon, \lambda}$-bounded; further $T\left(S_{1}(1)\right)$ is $\mathscr{T}_{\epsilon, \lambda}$-bounded since $T$ is a continuous linear operator. Besides, $\widetilde{I}_{A} \cdot T\left(S_{1}(1)\right)+$ $\tilde{I}_{A^{c}} \cdot T\left(S_{1}(1)\right) \subset T\left(S_{1}(1)\right)$ for all $A \in \mathscr{F}$ since $S_{1}(1)$ has this property and $T$ is a module homomorphism. Then $T\left(S_{1}(1)\right)$ is a.s. bounded; namely, $\xi:=\vee\left\{\|T(x)\|_{2}: x \in S_{1}(1)\right\} \in L_{+}^{0}(\mathscr{F})$ by Propositions 16 and 17. Since $1 /\left(\|x\|_{1}+(1 / n)\right) \cdot x \in S_{1}(1)$, for all $x \in S_{1}$ and $n \in N,\left\|T\left(1 /\left(\|x\|_{1}+(1 / n)\right) \cdot x\right)\right\|_{2} \leq \xi$, which implies that $\|T(x)\|_{2} \leq \xi \cdot\|x\|_{1}$, for all $x \in S_{1}$; that is to say, $T$ is a.s. bounded, at which time it is also clear that $\|T\|=\vee\left\{\|T(x)\|_{2}: x \in S_{1}(1)\right\}$.

(2) Necessity. From the proof of necessity of (1), if $T$ is a.s. bounded then $T$ is a module homomorphism. The fact that $T$ is a.s. bounded also obviously implies that $T$ is continuous from $\left(S_{1}, \mathscr{T}_{c}\right)$ to $\left(S_{2}, \mathscr{T}_{c}\right)$.

Sufficiency. Since $S_{2}(1):=\left\{y \in S_{2} \mid\|y\| \leq 1\right\}$ is a $\mathscr{T}_{c^{-}}$ neighborhood of the null element of $S_{2}$ there exists some $\epsilon \epsilon$ $L_{++}^{0}(\mathscr{F})$ such that $T\left(U_{\theta}(\epsilon)\right) \subset S_{2}(1)$, where $U_{\theta}(\epsilon)=\left\{x \in S_{1} \mid\right.$ $\left.\|x\|_{1} \leq \epsilon\right\}$. Thus for any $x \in S_{1},\left\|T\left(1 /\left(\|x\|_{1}+(1 / n)\right) \cdot x\right)\right\|_{2} \leq 1$, for all $x \in S_{1}$, and $n \in N$; namely, $\|T(x)\|_{2} \leq(1 / \epsilon)\left(\|x\|_{1}+\right.$ $(1 / n))$ by the fact that $T$ is a module homomorphism, which shows that $\|T(x)\|_{2} \leq(1 / \epsilon)\|x\|_{1}$, for all $x \in S_{1}$; namely, $T$ is a.s. bounded.

Remark 22. (1) of Theorem 21 was independently obtained by Guo in [5] and Haydon et al. in [6] although it is stated in a different way in [6, Proposition 5.6], one careful reader can see that Proposition 5.6 of [6] exactly amounts to (1) of Theorem 21. Our proof is different from Haydon et al.s in that we make use of something from the theory of MengerPN spaces (see the proof of Proposition 16) and our method may also be used in the proofs of some important results of Section 3.

Remark 23. Let $\left(S_{1},\|\cdot\|_{1}\right)$ and $\left(S_{2},\|\cdot\|_{2}\right)$ be two RN modules over $K$ with base $(\Omega, \mathscr{F}, \mu)$; when $T$ is a continuous module homomorphism from $\left(S_{1}, \mathscr{T}_{\epsilon, \lambda}\right)$ to $\left(S_{2}, \mathscr{T}_{\epsilon, \lambda}\right)$ or from $\left(S_{1}, \mathscr{T}_{c}\right)$ to $\left(S_{2}, \mathscr{T}_{c}\right)$, the process of proof of Theorem 21 has implied that $\|T\|=\vee\left\{\|T(x)\|_{2} \mid x \in S_{1}(1)\right\}$; further we have $N_{T}=$ $D_{T\left(S_{1}(1)\right)}$ by Proposition 16, where $N_{T}$ is the probabilistic norm of $T$, namely, the distribution function of $\|T\|$, and $D_{T\left(S_{1}(1)\right)}$ is the probabilistic diameter of $T\left(S_{1}(1)\right)$.

The proof of Proposition 24 below is similar to that of (1) of Theorem 21, so is omitted, but this proposition is very useful in the proof of the resonance theorem in Section 3 of this paper; we state it as follows.

Proposition 24 (see [14]). Let $(S,\|\cdot\|)$ be an $R N$ module over $K$ with base $(\Omega, \mathscr{F}, \mu)$ and $f: S \rightarrow L_{+}^{0}(\mathscr{F})$ such that the following two conditions are satisfied:

(1) $f(\alpha x)=\alpha \cdot f(x)$, for all $x \in S$ and all nonnegative numbers $\alpha$;

(2) $f(x+y) \leq f(x)+f(y)$, for all $x, y \in S$.

Then $f$ is a.s. bounded; namely, there is some $\xi \in L_{+}^{0}(\mathscr{F})$ such that $f(x) \leq \xi\|x\|$, for all $x \in S$, if and only if $f$ is continuous from $\left(S, \mathscr{T}_{\epsilon, \lambda}\right)$ to $\left(L^{0}(\mathscr{F}), \mathscr{T}_{\epsilon, \lambda}\right)$ and $f(\eta x)=$ $\eta \cdot f(x)$, for all $x \in S$ and $\eta \in L_{+}^{0}(\mathscr{F})$, at which time $\|f\|=\vee\{f(x) \mid x \in S(1)\}$, where $\|f\|=\wedge\left\{\xi \in L_{+}^{0}(\mathscr{F}) \mid\right.$ $f(x) \leq \xi\|x\|, \forall x \in S\}$; furthermore if, in addition, $(\Omega, \mathscr{F}, \mu)$ is a probability space, then $N_{f}$ (the distribution function of $\|f\|)=D_{f(S(1))}$.

It is well known that $B\left(S_{1}, S_{2}\right)$ is a Banach space when $S_{1}$ and $S_{2}$ are normed spaces and $S_{2}$ is complete. Similarly, $B\left(S_{1}, S_{2}\right)$ is $\mathscr{T}_{\epsilon, \lambda}$-complete when $\left(S_{1},\|\cdot\|_{1}\right)$ and $\left(S_{2},\|\cdot\|_{2}\right)$ are RN modules and $S_{2}$ is $\mathscr{T}_{\epsilon, \lambda}$-complete, which is independently pointed out by Guo in $[5,14]$ and Haydon et al. in [6]; in particular $S^{*}$ is $\mathscr{T}_{\epsilon, \lambda}$-complete for every $\mathrm{RN}$ module $S$. In fact, a more general result is proved in [14], namely, the following.

Proposition 25 (see [14]). Let $\left(S_{1},\|\cdot\|_{1}\right)$ and $\left(S_{2},\|\cdot\|_{2}\right)$ be two $R N$ spaces over $K$ with base $(\Omega, \mathscr{F}, \mu)$ such that $S_{2}$ is $\mathscr{T}_{\epsilon, \lambda^{-}}$ complete; then $B\left(S_{1}, S_{2}\right)$ is $\mathscr{T}_{\epsilon, \lambda}$-complete.

When $S_{1}$ and $S_{2}$ are both RN modules, since $\|T\|=$ $\vee\left\{\|T(x)\|_{2} \mid x \in S_{1}(1)\right\}$, for any $T \in B\left(S_{1}, B_{2}\right)$, the proof of Proposition 25 is similar to that of the classical case. But when $S_{1}$ and $S_{2}$ are only RN spaces, its proof needs Lemma 26 below. To state it, let us recall the canonical embedding mapping $J$ from an $\mathrm{RN}$ space $(S,\|\cdot\|)$ to $\left(S^{* *},\|\cdot\|^{* *}\right)$, where $S^{* *}=\left(S^{*}\right)^{*}, J(x)$ is defined by $(J(x))(f)=f(x)$, for all $f \in S^{*}$ and $x \in S$. It is easy to see that $J$ is random-norm preserving. As usual, $S$ is said to be random reflexive if $J$ is surjective. Generally, the $\mathscr{T}_{\epsilon, \lambda}$-closed submodule generated by $J(S)$ in $S^{* *}$ is called the $\mathscr{T}_{\epsilon, \lambda}$-closed submodule generated by $S$, denoted by $M(S)$; it is, obviously, a $\mathscr{T}_{\epsilon, \lambda}$-complete RN module.

Lemma 26 below is given and proved in [14]; here we give it a better proof.

Lemma 26 (see [14]). Let $\left(S_{1},\|\cdot\|_{1}\right)$ and $\left(S_{2},\|\cdot\|_{2}\right)$ be two $R N$ spaces over $K$ with base $(\Omega, \mathscr{F}, \mu)$ such that $S_{2}$ is $\mathscr{T}_{\epsilon, \lambda^{-}}$ complete. Then $B\left(S_{1}, B_{2}\right)$ is isomorphic to a $\mathscr{T}_{\epsilon, \lambda}$-closed subspace of $B\left(M\left(S_{1}, M\left(S_{2}\right)\right)\right.$ in a random-norm-preserving way.

Proof. Define $L: B\left(S_{1} \cdot S_{2}\right) \rightarrow B\left(M\left(S_{1}\right), M\left(S_{2}\right)\right)$ by $L(T)=$ $\left.T^{* *}\right|_{M\left(S_{1}\right)}$, where $T^{* *}$ is the random conjugate operator of $T^{*}$.

First, $L$ is well defined, namely, $L(T)\left(M\left(S_{1}\right)\right) \subset M\left(S_{2}\right)$, and isometric. Let $J_{1}: S_{1} \rightarrow S_{1}^{* *}$ and $J_{2}: S_{2} \rightarrow S_{2}^{* *}$ be the corresponding canonical embedding mappings; it is easy to check that $T^{* *} \circ J_{1}=J_{2} \circ T$, which not only shows that $T^{* *}\left(J_{1}\left(S_{1}\right)\right) \subset J_{2}\left(S_{2}\right)$ but also shows that $\|T\|=\left\|\left.T^{* *}\right|_{J_{1}\left(S_{1}\right)}\right\| \leq$ $\|L(T)\| \leq\left\|T^{* *}\right\|$. Since $\|T\|=\left\|T^{* *}\right\|,\|L(T)\|=\|T\|$. Further by (1) of Theorem 21 one can easily see that $L(T)\left(M\left(S_{1}\right)\right) \subset$ $M\left(S_{2}\right)$.

Second, $L\left(B\left(S_{1}, S_{2}\right)\right)$ is a $\mathscr{T}_{\epsilon, \lambda}$-closed subspace of $B\left(M\left(S_{1}\right), M\left(S_{2}\right)\right)$. Let $\left\{T_{n}, n \in N\right\}$ be a sequence in $B\left(S_{1}, S_{2}\right)$ such that $\left\{L\left(T_{n}\right), n \in N\right\}$ converges in the $(\epsilon, \lambda)$-topology to some $\bar{T} \in B\left(M\left(S_{1}\right), M\left(S_{2}\right)\right)$. Then $\left\{T_{n}, n \in N\right\}$ is also $\mathscr{T}_{\epsilon, \lambda}$-Cauchy in $B\left(S_{1}, S_{2}\right)$. We can, without loss of generality, assume that $\left\{\left\|T_{n}\right\|, n \in N\right\}$ converges a.s. to some $\xi$. Define $T: S_{1} \rightarrow S_{2}$ by $T(x)=\mathscr{T}_{\epsilon, \lambda}-\lim _{n \rightarrow \infty} T_{n}(x)$, for all $x \in S_{1}$; then $T$ is well defined since $S_{2}$ is $\mathscr{T}_{\epsilon, \lambda}$-complete, and $T$ is a.s. bounded since $\|T(x)\|_{2} \leq \xi\|x\|$, for all $x \in S_{1}$. Finally, it is easy to check that $L(T)=\bar{T}$. 
Remark 27. Since $B\left(M\left(S_{1}\right), M\left(S_{2}\right)\right)$ is always $\mathscr{T}_{\epsilon, \lambda}$-complete, so is $B\left(S_{1}, S_{2}\right)$ when $S_{2}$ is $\mathscr{T}_{\epsilon, \lambda}$-complete by Lemma 26 .

\section{Some Basic Principles of Continuous Module Homomorphisms between Random Normed Modules}

The main purpose of this section is to generalize some classical basic principles such as the resonance theorem, open mapping theorem, closed graph theorem, and inverse operator theorem to the context of random normed modules. It turns out that the counterparts under the $(\epsilon, \lambda)$-topology are consequences of the corresponding classical theorems on ordinary operators between quasinormed spaces except for the proof of the resonance theorem which is somewhat complicated. However, the counterparts under the locally $L^{0}$ convex topology are another thing since the usual reasoning fails to be valid; for example, the Baire category argument is no longer valid. Owing to the relations established in [8], we can prove them by converting their proofs to the case for the $(\epsilon, \lambda)$-topology.

The following surprisingly general uniform boundedness theorem is known (see [18]). But for the sake of reader's convenience, we state it as follows.

Proposition 28 (see [18]). Let $X$ be a linear topological space over $K$ of second category and $(Y,|\|\cdot\||)$ a quasinormed linear space. Let $\left\{T_{\alpha}, \alpha \in \wedge\right\}$ be a family of continuous mappings from $X$ to $Y$ such that the following three properties are satisfied:

(1) $\left|\left\|T_{\alpha}(x+y)\right\|\right| \leq\left|\left\|T_{\alpha}(x)\right\|\right|+\left|\left\|T_{\alpha}(y)\right\|\right|$, for all $x, y \in X$ and $\alpha \in \wedge$;

(2) $\left|\left\|T_{\alpha}(a x)\right\|\right|=\left|\left\|a T_{\alpha}(x)\right\|\right|$, for all $x \in X, \alpha \in \wedge$, and $a \geq 0$

(3) $\left\{T_{\alpha}(x), \alpha \in \wedge\right\}$ is bounded with respect to the linear topology induced by $|\|\cdot\||$ for each $x \in X$.

Then $\lim _{x \rightarrow \theta} T_{\alpha}(x)=\theta$ uniformly in $\alpha \in \wedge$.

Theorem 29. Let $\left(S_{1},\|\cdot\|_{1}\right)$ and $\left(S_{2},\|\cdot\|_{1}\right)$ be two RN modules over $K$ with base $(\Omega, \mathscr{F}, \mu)$ such that $S_{1}$ is $\mathscr{T}_{\epsilon, \lambda}$-complete. Let $\left\{T_{\alpha}: \alpha \in \wedge\right\}$. be a family of continuous module homomorphisms from $\left(S_{1}, \mathscr{T}_{\epsilon, \lambda}\right)$ to $\left(S_{2}, \mathscr{T}_{\epsilon, \lambda}\right)$. Then, one has the following:

(1) $\left\{T_{\alpha}: \alpha \in \wedge\right\}$ is $\mathscr{T}_{\epsilon, \lambda}$-bounded in $B\left(S_{1}, S_{2}\right)$ if and only if $\left\{T_{\alpha}(x): \alpha \in \wedge\right\}$ is $\mathscr{T}_{\epsilon, \lambda}$-bounded in $S_{2}$ for each $x \in S_{1}$;

(2) $\left\{T_{\alpha}: \alpha \in \wedge\right\}$ is a.s. bounded in $B\left(S_{1}, S_{2}\right)$ if and only if $\left\{T_{\alpha}(x): \alpha \in \wedge\right\}$ is a.s. bounded in $S_{2}$ for each $x \in S_{1}$.

Proof. We can, without loss of generality, assume that $(\Omega, \mathscr{F}, \mu)$ is a probability space.

(1) Necessity. Let $\left\{T_{\alpha}: \alpha \in \wedge\right\}$ be $\mathscr{T}_{\epsilon, \lambda}$-bounded in $B\left(S_{1}, S_{2}\right)$; namely, $D_{\left\{T_{\alpha}: \alpha \in \wedge\right\}} \in D^{+}$. For each $x \in S_{1}$, since $\left\|T_{\alpha}(x)\right\|_{2} \leq$ $\left\|T_{\alpha}\right\|\|x\|_{1}$, for all $\alpha \in \wedge, \mu\left\{\omega \in \Omega \quad\left\|T_{\alpha}(x)\right\|_{2}(\omega)<\right.$ $t\} \geq \mu\left(\left\{\omega \in \Omega \mid\left\|T_{\alpha}\right\|_{2}(\omega)<2 \sqrt{t}\right\} \cap\{\omega \in \Omega \mid\right.$ $\left.\left.\|x\|_{1}(\omega)<(1 / 2) \sqrt{t}\right\}\right) \geq \mu\left\{\omega \in \Omega \mid\left\|T_{\alpha}\right\|_{2}(\omega)<2 \sqrt{t}\right\}+$ $\mu\left\{\omega \in \Omega \mid\|x\|_{1}(\omega)<(1 / 2) \sqrt{t}\right\}-1$; namely, $N_{T_{\alpha}(x)}(t) \geq$ $N_{T_{\alpha}}(2 \sqrt{t})+N_{x}((1 / 2) \sqrt{t})-1$, for all $\alpha \in \wedge$ and $t>0$. Then, $D_{\left\{T_{\alpha}(x): \alpha \in \wedge\right\}}(t) \geq W\left(D_{\left\{T_{\alpha}: \alpha \in \wedge\right\}}(2 \sqrt{t}), N_{x}((1 / 2) \sqrt{t})\right)$, for all $x \in S_{1}$ and $t>0$, where $W(a, b)=\max (a+b-1,0)$, for all $a, b \in[0,1]$. Since $D_{\left\{T_{\alpha}: \alpha \in \wedge\right\}} \in D^{+}$and $N_{x} \in D^{+}$for any $x \in S_{1}$, then $D_{\left\{T_{\alpha}(x): \alpha \in \wedge\right\}} \in D^{+}$; namely, $\left\{T_{\alpha}(x): \alpha \in \wedge\right\}$ is $\mathscr{T}_{\epsilon, \lambda}$-bounded in $S_{2}$ for each $x \in S_{1}$.

Sufficiency. Let $|\|\cdot\||: S_{2} \rightarrow[0,+\infty)$ be defined by $|\|y\||=$ $\int_{\Omega}(\|y\| /(1+\|y\|)) d \mu$ for any $y \in S_{2}$; then $\left(S_{2},|\|\cdot\||\right)$ is a quasinormed linear space and $|\|\cdot\||$ induces the $(\epsilon, \lambda)$-topology for $S_{2}$. Since $\left(S_{1}, \mathscr{T}_{\epsilon, \lambda}\right)$ is a linear topological space of the second category and it is also clear that $\left\{T_{\alpha}: \alpha \in \wedge\right\}$ satisfies

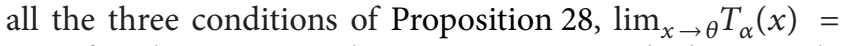
0 uniformly in $\alpha \in \wedge$ by Proposition 28, which certainly implies that $\bigcup_{\alpha \in \wedge} T_{\alpha}(A)$ is $\mathscr{T}_{\epsilon, \lambda}$-bounded in $S_{2}$ for each $\mathscr{T}_{\epsilon, \lambda^{-}}$ bounded set $A$ in $S_{1}$, in particular $\bigcup_{\alpha \in \wedge} T_{\alpha}\left(S_{1}(1)\right)$ is $\mathscr{T}_{\epsilon, \lambda} \lambda^{-}$ bounded. By Remark 23, $N_{T_{\alpha}}=D_{T_{\alpha}\left(S_{1}(1)\right)}$, for all $\alpha \in \wedge$. Define $F:[-\infty,+\infty] \rightarrow[0,1]$ by $F(t)=\inf _{\alpha \in \wedge} D_{T_{\alpha}\left(S_{1}(1)\right)}(t)$, for all $t \in[-\infty,+\infty]$, and $l^{-} F:[-\infty,+\infty]$ by $\left(l^{-} F\right)(-\infty)=$ $0,\left(l^{-} F\right)(+\infty)=1$, and $\left(l^{-} F\right)(t)=\sup \left\{F\left(t^{\prime}\right) \mid t^{\prime}<t\right\}$, for all $t \in(-\infty,+\infty)$; denote $\bigcup_{\alpha \in \Lambda} T_{\alpha}\left(S_{1}(1)\right)$ by $A$; then one can easily check that $D_{A}=l^{-} F=D_{\left\{T_{\alpha}: \alpha \in \wedge\right\}}$; then $\left\{T_{\alpha}: \alpha \in \wedge\right\}$ is $\mathscr{T}_{\epsilon, \lambda}$-bounded since $D_{A} \in D^{+}$.

(2) Necessity of (2) Is Clear. We prove sufficiency of (2) as follows.

Denote the family of finite subsets of $\wedge$ by $\mathscr{F}(\wedge)$. For any $F \in \mathscr{F}(\wedge)$, define $f_{F}: S_{1} \rightarrow L_{+}^{0}(\mathscr{F})$ by $f_{F}(x)=\vee\left\{\left\|T_{\alpha} x\right\|_{2} \mid\right.$ $\alpha \in F\}$, for all $x \in S_{1}$; then $f_{F}$ is continuous from $\left(S_{1}, \mathscr{T}_{\epsilon, \lambda}\right)$ to $\left(L^{0}(\mathscr{F}, K), \mathscr{T}_{\epsilon, \lambda}\right)$ and $f_{F}(\xi x)=\xi \cdot f_{F}(x)$, for all $\xi \in L_{+}^{0}(\mathscr{F})$ and $x \in S_{1}$, and hence $f_{F}$ is a.s. bounded and $\left\|f_{F}\right\|=\vee\left\{f_{F}(x) \mid\right.$ $\left.x \in S_{1}(1)\right\}=\vee\left\{\left\|T_{\alpha}\right\|: \alpha \in F\right\}$. It is obvious that $\left\{\left\|f_{F}\right\| \mid F \in\right.$ $\mathscr{F}(\wedge)\}=\vee\left\{\left\|T_{\alpha}\right\| \mid \alpha \in \wedge\right\}$, so we only need to prove that $\left\{\left\|f_{F}\right\| \mid F \in \mathscr{F}(\wedge)\right\}$ is a.s. bounded, which is equivalent to the fact that $\left\{\left\|f_{F}\right\| \mid F \in \mathscr{F}(\wedge)\right\}$ is $\mathscr{T}_{\epsilon, \lambda}$-bounded in $L_{+}^{0}(\mathscr{F})$ by Proposition 16. Since $\vee\left\{f_{F}(x) \mid F \in \mathscr{F}(\wedge)\right\}=\vee\left\{\left\|T_{\alpha}(x)\right\| \mid\right.$ $\alpha \in \wedge\}$ for each $x \in S_{1},\left\{f_{F}(x) \mid F \in \mathscr{F}(\wedge)\right\}$ is a.s. bounded and hence also $\mathscr{T}_{\epsilon, \lambda}$-bounded for each $x \in S_{1}$. In the process of proof of sufficiency of (1), by replacing $S_{2}$ with $L^{0}(\mathscr{F}, K)$ and the same reasoning we have that $\left\{\left\|f_{F}\right\| \mid F \in \mathscr{F}(\wedge)\right\}$ is $\mathscr{T}_{\epsilon, \lambda^{-}}$bounded since $\left\{f_{F} \mid F \in \mathscr{F}(\wedge)\right\}$ still satisfies all the three conditions of Proposition 28.

Theorems 30,31 , and 32 below are essentially known since they can be regarded as a special case of the classical closed graph theorem, open mapping theorem, and inverse operator theorem between Fréchét spaces only by noticing that a $\mathscr{T}_{\epsilon, \lambda}$ complete RN space is a Fréchét space, but we would like to state them for the convenience of subsequent applications.

Theorem 30. Let $\left(S_{1},\|\cdot\|_{1}\right)$ and $\left(S_{2},\|\cdot\|_{2}\right)$ be $\mathscr{T}_{\epsilon, \lambda}$-complete $R N$ modules over $K$ with base $(\Omega, \mathscr{F}, \mu)$ and $T: S_{1} \rightarrow S_{2}$ a module homomorphism. Then $T$ is continuous from $\left(S_{1}, \mathscr{T}_{\epsilon, \lambda}\right)$ to $\left(S_{2}, \mathscr{T}_{\epsilon, \lambda}\right)$ if and only if $T$ is $\mathscr{T}_{\epsilon, \lambda}$-closed (namely, the graph of $T$ is $\mathscr{T}_{\epsilon, \lambda}$-closed in $S_{1} \times S_{2}$ ). 
Theorem 31. Let $\left(S_{1},\|\cdot\|_{1}\right)$ and $\left(S_{2},\|\cdot\|_{2}\right)$ be $\mathscr{T}_{\epsilon, \lambda}$-complete $R N$ modules over $K$ with base $(\Omega, \mathscr{F}, \mu)$ and $T$ a surjective continuous module homomorphism from $\left(S_{1}, \mathscr{T}_{\epsilon, \lambda}\right)$ to $\left(S_{2}, \mathscr{T}_{\epsilon, \lambda}\right)$. Then $T$ is $\mathscr{T}_{\epsilon, \lambda}$-open; namely, $T(G)$ is $\mathscr{T}_{\epsilon, \lambda}$-open for each $\mathscr{T}_{\epsilon, \lambda}$ - open subset $G$ of $S_{1}$.

Theorem 32. Let $\left(S_{1},\|\cdot\|_{1}\right)$ and $\left(S_{2},\|\cdot\|_{2}\right)$ be $\mathscr{T}_{\epsilon, \lambda}$-complete $R N$ modules over $K$ with base $(\Omega, \mathscr{F}, \mu)$ and $T$ a bijective continuous module homomorphism from $\left(S_{1}, \mathscr{T}_{\epsilon, \lambda}\right)$ to $\left(S_{2}, \mathscr{T}_{\epsilon, \lambda}\right)$. Then $T^{-1}$ is also a continuous module homomorphism from $\left(S_{2}, \mathscr{T}_{\epsilon, \lambda}\right)$ to $\left(S_{1}, \mathscr{T}_{\epsilon, \lambda}\right)$.

To give the versions of Theorems 29 up to 32 under the locally $L^{0}$-convex topology, let us first recall the notion of countable concatenation property of a set or an $L^{0}(\mathscr{F}, K)$ module. The introducing of the notion utterly results from the study of the locally $L^{0}$-convex topology, the reader will see that this notion is ubiquitous in the theory of the locally $L^{0}$-convex topology; From now on, we always suppose that all the $L^{0}(\mathscr{F}, K)$-modules $E$ involved in this paper have the property that for any $x, y \in E$, if there is a countable partition $\left\{A_{n}, n \in N\right\}$ of $\Omega$ to $\mathscr{F}$ such that $\widetilde{I}_{A_{n}} x=\widetilde{I}_{A_{n}} y$ for each $n \in N$, then $x=y$. Guo already pointed out in [8] that all random locally convex modules possess this property, so the assumption is not too restrictive.

Definition 33 (see [8]). Let $S$ be an $L^{0}(\mathscr{F}, K)$-module. A subset $G$ of $S$ is said to have the countable concatenation property if for each sequence $\left\{g_{n}, n \in N\right\}$ in $G$ and each countable partition $\left\{A_{n}, n \in N\right\}$ of $\Omega$ to $\mathscr{F}$, there is $g \in G$ such that $\widetilde{I}_{A_{n}} g=\widetilde{I}_{A_{n}} g_{n}$, for all $n \in N$.

Two propositions below are key in this paper.

Proposition 34 (see [8]). Let (S, $\|\cdot\|)$ be an $R N$ module and $G$ a subset with the countable concatenation property. Then $\bar{G}_{\epsilon, \lambda}=$ $\bar{G}_{c}$, where $\bar{G}_{\epsilon, \lambda}$ and $\bar{G}_{c}$ stand for the closures of $G$ under the $(\epsilon, \lambda)$-topology and the locally $L^{0}$-convex topology, respectively.

Proposition 35 (see [8]). An RN module $(S,\|\cdot\|)$ is $\mathscr{T}_{\epsilon, \lambda^{-}}$ complete if and only if it is $\mathscr{T}_{c}$-complete and $S$ has the countable concatenation property.

Theorem 36 below has been used to establish random convex analysis; compare [12].

Theorem 36. Let $\left(S_{1},\|\cdot\|_{1}\right)$ and $\left(S_{2},\|\cdot\|_{2}\right)$ be two RN modules over $K$ with base $(\Omega, \mathscr{F}, \mu)$ such that $S_{1}$ is $\mathscr{T}_{c}$-complete and has the countable concatenation property. Let $\left\{T_{\alpha}: \alpha \in \wedge\right\}$ be a family of continuous module homomorphism from $\left(S_{1}, \mathscr{T}_{c}\right)$ to $\left(S_{2}, \mathscr{T}_{c}\right)$; then $\left\{T_{\alpha}: \alpha \in \wedge\right\}$ is $\mathscr{T}_{c}$-bounded if and only if $\left\{T_{\alpha}(x): \alpha \in \wedge\right\}$ is $\mathscr{T}_{c}$-bounded for each $x \in S_{1}$.

Proof. By Proposition 35, it follows from (2) of Theorem 29.

Theorem 37. Let $\left(S_{1},\|\cdot\|_{1}\right)$ and $\left(S_{2},\|\cdot\|_{2}\right)$ be two $\mathscr{T}_{c}$-complete $R N$ modules over $K$ with base $(\Omega, \mathscr{F}, \mu)$ such that $S_{1}$ and $S_{2}$ have the countable concatenation property. Then, a module homomorphism $T: S_{1} \rightarrow S_{2}$ is continuous from $\left(S_{1}, \mathscr{T}_{c}\right)$ to $\left(S_{2}, \mathscr{T}_{c}\right)$ if and only if $T$ is $\mathscr{T}_{c}$-closed (namely, the graph of $T$ is $\mathscr{T}_{c}$-closed in $S_{1} \times S_{2}$ ).

Proof. It is clear that the graph of $T$ has the countable concatenation property. By Theorem 21, $T$ is continuous from $\left(S_{1}, \mathscr{T}_{\epsilon, \lambda}\right)$ to $\left(S_{2}, \mathscr{T}_{\epsilon, \lambda}\right)$ if and only if it is continuous from $\left(S_{1}, \mathscr{T}_{c}\right)$ to $\left(S_{2}, \mathscr{T}_{c}\right)$. So, the proof follows from Propositions 34 and 35 and Theorem 30.

Theorem 38. Let $\left(S_{1},\|\cdot\|_{1}\right)$ and $\left(S_{2},\|\cdot\|_{2}\right)$ be two $\mathscr{T}_{c^{-}}$ complete $R N$ modules over $K$ with base $(\Omega, \mathscr{F}, \mu)$ such that $S_{1}$ and $S_{2}$ have the countable concatenation property. If $T$ : $S_{1} \rightarrow S_{2}$ is a bijective continuous module homomorphism from $\left(S_{1}, \mathscr{T}_{c}\right)$ to $\left(S_{2}, \mathscr{T}_{c}\right)$, then $T^{-1}$ is also continuous module homomorphism from $\left(S_{2}, \mathscr{T}_{c}\right)$ to $\left(S_{1}, \mathscr{T}_{c}\right)$.

Proof. It follows from Proposition 35 and Theorems 21 and 32.

To give Theorem 40 below, we need Lemma 39 below.

Lemma 39. Let $(S,\|\cdot\|)$ be a $\mathscr{T}_{c}$-complete $R N$ module over $K$ with base $(\Omega, \mathscr{F}, \mu)$ and $M$ a $\mathscr{T}_{c}$-closed submodule of $S$ such that both $S$ and $M$ have the countable concatenation property. Then, $\left(S / M,\|\cdot\|_{M}\right)$ is still a $\mathscr{T}_{c}$-complete $R N$ module and $S / M$ has the countable concatenation property, where $S / M$ is the quotient module of $S$ with respect to $M$ and $\|\cdot\|_{M}: S / M \rightarrow$ $L_{+}^{0}(\mathscr{F})$ is defined by $\|x+M\|_{M}=\wedge\{\|y\| \mid y \in x+M\}$.

Proof. By Proposition 35 both $S$ and $M$ are $\mathscr{T}_{\epsilon, \lambda}$-complete; then $\left(S / M,\|\cdot\|_{M}\right)$ is a $\mathscr{T}_{\epsilon, \lambda}$-complete $\mathrm{RN}$ module by the theory of quotient spaces for Fréchét spaces. The proof again follows from Proposition 35.

Theorem 40. Let $\left(S_{1},\|\cdot\|_{1}\right)$ and $\left(S_{2},\|\cdot\|_{2}\right)$ be two $\mathscr{T}_{c^{-}}$ complete $R N$ modules over $K$ with base $(\Omega, \mathscr{F}, \mu)$ such that $S_{1}$ and $S_{2}$ have the countable concatenation property. If $T$ is a surjective continuous module homomorphism from $\left(S_{1}, \mathscr{T}_{c}\right)$ to $\left(S_{2}, \mathscr{T}_{c}\right)$, then $T$ is $\mathscr{T}_{c}$-open; namely, $T(G)$ is $\mathscr{T}_{c}$-open for each $\mathscr{T}_{c}$-open subset $G$ of $S_{1}$.

Proof. Let $M=\left\{x \in S_{1} \mid T(x)=0\right\}$; then $M$ is $\mathscr{T}_{c^{-}}$ closed and has the countable concatenation property. Define $\widehat{T}:\left(S_{1} / M,\|\cdot\|_{M}\right) \rightarrow S_{2}$ by $\widehat{T}(x+M)=T(x)$, for all $x \in S_{1}$, where $\left(S_{1} / M,\|\cdot\|_{M}\right)$ is the quotient space of $\left(S_{1},\|\cdot\|_{1}\right)$ with respect to $M$; it is clear that $\widehat{T}$ is a bijective continuous module homomorphism from $\left(S_{1} / M, \mathscr{T}_{c}\right)$ to $\left(S_{2}, \mathscr{T}_{c}\right)$. By Theorem 38, $\widehat{T}^{-1}$ is a continuous module homomorphism from $\left(S_{2}, \mathscr{T}_{c}\right)$ to $\left(S_{1} / M, \mathscr{T}_{c}\right)$. So, $\widehat{T}(\widehat{G})$ is a $\mathscr{T}_{c}$-open subset in $S_{2}$ for each $\mathscr{T}_{c}$-open subset $\widehat{G}$ of $S_{1} / M$. Observing that $T=\widehat{T} \circ J$, where $J: S_{1} \rightarrow S_{1} / M$ is the canonical quotient mapping, then $T$ is $\mathscr{T}_{c}$-open.

Remark 41. Since a $\mathscr{T}_{c}$-complete $\mathrm{RN}$ module is not necessarily of second category, we can not obtain Theorem 40 by using the Baire category argument which is used in the proof of the classical open mapping theorem. In fact, the proof 
of Theorem 40 also gives a new proof of the classical open mapping theorem.

\section{The Orthogonal Decomposition Theorem and Riesz Representation Theorem in Complete Random Inner Product Modules under the Two Kinds of Topologies}

The orthogonal decomposition theorem in complete random inner product modules was already pointed out in $[3,20]$ without a detailed proof since it can be indirectly and similarly obtained from a best approximation result of $[5,21]$ in a special complete random inner product module. Here, we give it a detailed proof. The Riesz representation theorem in complete random inner product modules was proved in [20], but we did not strictly distinguish, by symbols, between measurable functions and their equivalence classes, so the readability of the proof given in [20] is not very good. Here, we also give a new proof for the sake of convenience for readers; the idea is, of course, due to [20].

Definition 42 (see [3]). An ordered pair $(S,\langle\cdot, \cdot\rangle)$ is called a random inner product space (briefly, an RIP space) over $K$ with base $(\Omega, \mathscr{F}, \mu)$ if $S$ is a linear space over $K$ and $\langle\cdot, \cdot\rangle$ is a mapping from $S \times S \rightarrow L^{0}(\mathscr{F}, K)$ such that the following are satisfied:

(RIP-1) $\langle x, x\rangle \in L_{+}^{0}(\mathscr{F})$ and $\langle x, x\rangle=0$ implies $x=0$ (the null element of $S$ );

(RIP-2) $\langle\alpha \cdot x, y\rangle=\alpha \cdot\langle x, y\rangle$, for all $\alpha \in K$ and $x, y \in S$;

(RIP-3) $\langle x, y\rangle=\overline{\langle y, x\rangle}$, for all $x, y \in S$, where $\overline{\langle y, x\rangle}$ stands for the complex conjugation of $\langle y, x\rangle$;

(RIP-4) $\langle x+y, z\rangle=\langle x, z\rangle+\langle y, z\rangle$, for all $x, y, z \in S$,

where $\langle x, y\rangle$ is called the random inner product of $x$ and $y$ in $S$.

Furthermore, if, in addition, $S$ is an $L^{0}(\mathscr{F}, K)$-module and the following is satisfied:

(RIPM-1) $\langle\xi \cdot x, y\rangle=\xi \cdot\langle x, y\rangle$, for all $\xi \in L^{0}(\mathscr{F}, K)$ and $x, y \in S$,

then $(S,\langle\cdot, \cdot\rangle)$ is called a random inner product module (briefly, an RIP module) over $K$ with base $(\Omega, \mathscr{F}, \mu)$, at which time $\langle x, y\rangle$ is called the $L^{0}$-inner product of $x$ and $y$ in $S$; namely, an $L^{0}$-inner product is a random inner product with the property (RIPM-1).

In an RIP space $(S,\langle\cdot, \cdot\rangle), x$ is orthogonal to $y$, denoted by $x \perp y$, if $\langle x, y\rangle=0$. For a subset $M$ of $S, M^{\perp}:=\{y \in$ $S \mid\langle x, y\rangle=0, \forall x \in M\}$ is the orthogonal complement of $M$ in $S$. Define $\|\cdot\|: S \rightarrow L_{+}^{0}(\mathscr{F})$ by $\|x\|=\sqrt{\langle x, x\rangle}$, for all $x \in S$; then $(S,\|\cdot\|)$ is an RN space over $K$ with base $(\Omega, \mathscr{F}, \mu)$ by the following random Schwartz inequality (namely, Lemma 43 below); $\|\cdot\|$ is the random norm derived from $\langle\cdot, \cdot\rangle$. It is also clear that $(S,\|\cdot\|)$ is an RN module if $(S,\langle\cdot, \cdot\rangle)$ is an RIP module.
Lemma 43. Let $(S,\langle\cdot, \cdot\rangle)$ be an RIP space over $K$ with base $(\Omega, \mathscr{F}, \mu)$. Then $|\langle x, y\rangle| \leq\|x\| \cdot\|y\|$, for all $x, y \in S$.

Proof. Let $x$ and $y$ be fixed and then choose $\langle x, y\rangle^{0},\|x\|^{0}$, and $\|y\|^{0}$ as given representatives of $\langle x, y\rangle,\|x\|$, and $\|y\|$, respectively. Since $\langle\alpha x+y, \alpha x+y\rangle=|\alpha|^{2}\|x\|^{2}+2 \operatorname{Re}(\alpha$. $\langle x, y\rangle)+\|y\|^{2} \geq 0$, for all $\alpha \in K$. Let $K(1)=\{\beta \in K|| \beta \mid=1\}$, then taking $\alpha=t \beta$ with $t \in R$ and $\beta \in K(1)$ yields that $t^{2} \cdot\|x\|^{2}+2 t \cdot \operatorname{Re}(\beta \cdot\langle x, y\rangle)+\|y\|^{2} \geq 0$, for all $t \in R$ and $\beta \in K(1)$; namely, $t^{2} \cdot\left(\|x\|^{0}(\omega)\right)^{2}+2 t \cdot \operatorname{Re}\left(\beta \cdot\langle x, y\rangle^{0}(\omega)\right)+$ $\left(\|y\|^{0}(\omega)\right)^{2} \geq 0$ a.s., for all $t \in R$ and $\beta \in K(1)$. Since $R$ and $K(1)$ are separable, we can obtain an $\mathscr{F}$-measurable $\Omega_{0}$ with $\mu\left(\Omega \backslash \Omega_{0}\right)=0$ such that $t^{2} \cdot\left(\|x\|^{0}(\omega)\right)^{2}+2 t \cdot \operatorname{Re}\left(\beta \cdot\langle x, y\rangle^{0}(\omega)\right)+$ $\left(\|y\|^{0}(\omega)\right)^{2} \geq 0$ on $\Omega_{0}$, for all $t \in R$ and $\beta \in K(1)$.

For each $\omega \in \Omega_{0}$, we can always take $\beta \in K(1)$ such that $\beta \cdot\langle x, y\rangle^{0}(\omega)=\left|\langle x, y\rangle^{0}(\omega)\right|$; then we have that $t^{2} \cdot\left(\|x\|^{0}(\omega)\right)^{2}+$ $2 t \cdot\left|\langle x, y\rangle^{0}(\omega)\right|+\left(\|y\|^{0}(\omega)\right)^{2} \geq 0$ on $\Omega_{0}$, for all $t \in R$, so $\left|\langle x, y\rangle^{0}(\omega)\right| \leq\|x\|^{0}(\omega) \cdot\|y\|^{0}(\omega)$, for all $\omega \in \Omega_{0}$; namely, $|\langle x, y\rangle| \leq\|x\| \cdot\|y\|$.

Remark 44. In the proof of Lemma 43, we use a technique, namely, making use of separability of the scalar field $K$, which was first used in the proof of extension theorem for complex random linear functionals; compare $[2,8]$.

Lemma 45. Let $(S,\langle\cdot, \cdot\rangle)$ be an RIP space over $K$ with base $(\Omega, \mathscr{F}, \mu), M$ a subspace of $M$, and $x_{0} \in M$. Then $\left\|x-x_{0}\right\|=$ $\wedge\{\|x-y\|: y \in M\}$ if and only if $x-x_{0} \perp M$.

Proof. Sufficiency is obvious. As for the necessity, since $\left\|x-x_{0}\right\|^{2} \leq\left\|x-x_{0}-\alpha y\right\|^{2}$, for all $\alpha \in K$ and $y \in M$, namely, $2 \operatorname{Re}\left(\alpha\left\langle y, x-x_{0}\right\rangle\right) \leq|a|^{2}\|y\|^{2}$, taking $\alpha=(1 / n) a$ yields that $2 \operatorname{Re}\left(a\left\langle y, x-x_{0}\right\rangle\right) \leq(1 / n)|a|^{2}\|y\|^{2}$, which implies that $\operatorname{Re}\left(a\left\langle y, x-x_{0}\right\rangle\right) \leq 0$, for all $a \in K$ and $y \in M$. Similar to the proof of Lemma 43 , one can have that $\left\langle y, x-x_{0}\right\rangle=0$, for all $y \in M$.

Remark 46. $x_{0}$ in Lemma 45 is called a best approximation point of $x$ in $M$; such a kind of idea was earlier used in $[5,21-23]$ for the study of best approximation problems in Lebesgue-Bochner function spaces.

Theorem 47. Let $(S,\langle\cdot, \cdot\rangle)$ be a $\mathscr{T}_{\epsilon, \lambda}$-complete RIP module over $K$ with base $(\Omega, \mathscr{F}, \mu)$ and $M a \mathscr{T}_{\epsilon, \lambda}$-closed subspace of $S$. Then $S=M \oplus M^{\perp}$ if and only if $M$ is a submodule.

Proof.

Sufficiency. For each $x \in S$, let $d(x, M)=\wedge\{\|x-y\| \mid y \in M\}$. By Proposition 17 there exists a sequence $\left\{y_{n}, n \in N\right\}$ in $M$ such that $\left\{\left\|x-y_{n}\right\|, n \in N\right\}$ converges a.s. to $d(x, M)$ in a nonincreasing manner. Similar to the classical case, one can deduce that $\left\{y_{n}, x \in N\right\}$ is a $\mathscr{T}_{\epsilon, \lambda}$-Cauchy sequence and hence convergent to some $x_{0} \in M$ such that $\left\|x-x_{0}\right\|=d(x, M)$. By Lemma $45, x-x_{0} \perp M$. Hence, each $x \in S$ can be written as $x-x_{0}+x_{0} \in M^{\perp} \oplus M$.

Necessity. We only need to prove that $\widetilde{I}_{A} x \in M$ for each $A \in \mathscr{F}$ and $x \in M$. Let $\widetilde{I}_{A} x=x_{1}+x_{2}$ with $x_{1} \in M$ and $x_{2} \in M^{\perp}$; since $x_{2} \perp \widetilde{I}_{A} x$ implies $x_{2}=\theta, \widetilde{I}_{A} x=x_{1} \in M$. 
Theorem 48. Let $(S,\langle\cdot, \cdot\rangle)$ be a $\mathscr{T}_{\epsilon, \lambda}$-complete RIP module over $K$ with base $(\Omega, \mathscr{F}, \mu)$. Then for each $f \in S^{*}$ there exists a unique $y_{f} \in S$ such that $f(x)=\left\langle x, y_{f}\right\rangle$, for all $x \in S$, and $\|f\|=\left\|y_{f}\right\|$.

Before the proof of Theorem 48, let us first introduce some notation and terminology as follows. Let $\xi$ be in $L^{0}(\mathscr{F}, K)$ with a chosen representative $\xi^{0} .\left(\xi^{0}\right)^{-1}: \Omega \rightarrow K$ is defined by $\left(\xi^{0}\right)^{-1}(\omega)=1 / \xi^{0}(\omega)$ if $\xi^{0}(\omega) \neq 0$ and 0 otherwise. Then the equivalence class determined by $\left(\xi^{0}\right)^{-1}$ is called the generalized inverse of $\xi$, denoted by $\xi^{-1}$, and $|\xi|^{-1} \xi$ is called the sign of $\xi$, denoted by $\operatorname{sgn}(\xi)$. It is obvious that $\xi \cdot \xi^{-1}=\widetilde{I}_{A}$, where $A=\left\{\omega \in \Omega \mid \xi^{0}(\omega) \neq 0\right\}$, and $\overline{\operatorname{sgn}(\xi)} \cdot \xi=|\xi|$. Besides, for any $\xi$ and $\eta$ in $L^{0}(\mathscr{F}),[\xi \leq \eta]$ denotes the equivalence class of the $\mathscr{F}$-measurable set $\left\{\omega \in \Omega \mid \xi^{0}(\omega) \leq \eta^{0}(\omega)\right\}$, where $\xi^{0}$ and $\eta^{0}$ are any chosen representatives of $\xi$ and $\eta$, respectively. Similarly, one can understand the meaning of $[\xi>\eta]$.

We can now prove Theorem 48.

Proof of Theorem 48. Let $\operatorname{Ran}(f)=\{f(x) \mid x \in S\}$ and $N(f)=\{x \in S \mid f(x)=0\}$; then $\operatorname{Ran}(f)$ is a submodule of $L^{0}(\mathscr{F}, K)$ and $N(f)$ a $\mathscr{T}_{\epsilon, \lambda}$-closed submodule of $S$.

By Proposition $17\{|f(x)| \mid x \in S\}$ is upward directed and hence there exists a sequence $\left\{x_{n}, n \in N\right\}$ in $S$ such that $\left\{\left|f\left(x_{n}\right)\right|, n \in N\right\}$ converges a.s. to $\xi:=\vee\{|f(x)| \mid x \in S\}$ in a nondecreasing manner.

Denote $\xi_{n}=\left|f\left(x_{n}\right)\right|$, for all $n \in N$; let $\xi^{0}$ and $\xi_{n}^{0}$ be any chosen representatives of $\xi$ and $\xi_{n}$, respectively, $B=\{\omega \in \Omega \mid$ $\left.\xi^{0}(\omega)>0\right\}$, and $B_{n}=\left\{\omega \in \Omega \mid \xi_{n}^{0}(\omega)>0\right\}$, for all $n \geq 1$. We can, without loss of generality, assume that $B_{n} \subset B_{n+1}$, for all $n \geq 1$, and $\bigcup_{n=1}^{\infty} B_{n}=B$. Further, let $B_{0}=\varnothing$ and $A_{n}=B_{n} \backslash B_{n-1}$, for all $n \geq 1$; then $A_{i} \cap A_{j}=\varnothing$, where $i \neq j$, and $B=\bigcup_{n=1}^{\infty} A_{n}$. Since $\xi_{n}^{0}>0$ on $A_{n}$, we have $f\left(z_{n}\right)=\widetilde{I}_{A_{n}}$, where $z_{n}=\widetilde{I}_{A_{n}}\left(f\left(x_{n}\right)\right)^{-1} \cdot x_{n}$, for all $n \geq 1$.

If $\mu(B)=0$, then taking $y_{f}=\theta$ ends the proof. If $\mu(B)>0$, then $f \neq 0$, in which case we can assume that $\mu\left(A_{n}\right)>0$, for all $n \in N$. By Theorem 47 there exists a unique $\bar{z}_{n} \in N(f)^{\perp}$ such that $z_{n}-\bar{z}_{n} \in N(f)$, and hence $f\left(\bar{z}_{n}\right)=f\left(z_{n}\right)=\widetilde{I}_{A_{n}}$, for all $n \in$ $N$. Since $\widetilde{I}_{A_{n}} x-\widetilde{I}_{A_{n}} f(x) \bar{z}_{n} \in N(f),\left\langle\widetilde{I}_{A_{n}} x-\widetilde{I}_{A_{n}} f(x) \bar{z}_{n}, \bar{z}_{n}\right\rangle=$ 0 , for all $n \in N$ and $x \in S$.

Since $\widetilde{I}_{A_{n}}=f\left(\bar{z}_{n}\right)=\left|f\left(\bar{z}_{n}\right)\right| \leq\|f\|\left\|\bar{z}_{n}\right\|,\left\|\bar{z}_{n}\right\|>0$ on $A_{n}$ and $\widetilde{I}_{A_{n}} f(x)=\left\langle x, z_{n}^{*}\right\rangle$, for all $x \in S$, where $z_{n}^{*}=$ $\widetilde{I}_{A_{n}}\left(\left\|\bar{z}_{n}\right\|\right)^{-1} \bar{z}_{n}$, for all $n \in N$. Let $y_{n}=\sum_{i=1}^{n} z_{i}^{*}$, for all $n \in N$. By noticing that $I_{B} f(x)=f(x)$, for all $x \in S$, and $\widetilde{I}_{B}=$ $\lim _{n \rightarrow \infty} \widetilde{I}_{B_{n}}=\lim _{n \rightarrow \infty} \sum_{i=1}^{n} \widetilde{I}_{A_{i}}, f(x)=\lim _{n \rightarrow \infty}\left(\widetilde{I}_{B_{n}} f(x)\right)=$ $\lim _{n \rightarrow \infty}\left(\sum_{i=1}^{n} \widetilde{I}_{A_{i}} f(x)\right)=\lim _{n \rightarrow \infty}\left\langle x, y_{n}\right\rangle$ (where convergence means the a.s. convergence). We can, without loss of generality, assume that $(\Omega, \mathscr{F}, \mu)$ is a probability space; then $\mu\left\{\omega \in \Omega \mid\left\|y_{n+m}-y_{n}\right\|(\omega)>\epsilon\right\} \leq \sum_{i=n+1}^{n+m} \mu\left(A_{i}\right)$, for any $\epsilon>0$ and $n, m \in N$, which implies that $\left\{y_{n}, n \in N\right\}$ is $\mathscr{T}_{\epsilon, \lambda}$-Cauchy and hence convergent to some $y_{f} \in S$, so $f(x)=\left\langle x, y_{f}\right\rangle$, for all $x \in S$.

$\|f\| \leq\left\|y_{f}\right\|$ is obvious. Now, let $A=\left[\left\|y_{f}\right\|>0\right]$ and $D=$ $\left[\left|f\left(y_{f}\right)\right| \leq\|f\|\left\|y_{f}\right\|\right]$; then $\mu(D)=1$ and $I_{A}\|f\| \geq I_{A}\left\|y_{f}\right\|$, where $I_{A}=\widetilde{I}_{A^{0}}$ with $A^{0}$ being any representative of $A$. On the other hand, $\left(1-I_{A}\right)\|f\| \geq 0=\left(1-I_{A}\right)\left\|y_{f}\right\|$, so $\|f\|=$ $I_{A}\|f\|+\left(1-I_{A}\right)\|f\| \geq I_{A}\left\|y_{f}\right\|+\left(1-I_{A}\right)\left\|y_{f}\right\|=\left\|y_{f}\right\|$. Finally, the uniqueness of $y_{f}$ is obvious.

The version of Theorem 48 under the locally $L^{0}$-convex topology, namely, Corollary 50 below, was given in [8]. Corollary 49 below is the version of Theorem 47 under the locally $L^{0}$-convex topology.

Corollary 49. Let $(S,\|\cdot\|)$ be a $\mathscr{T}_{c}$-complete RIP module over $K$ with base $(\Omega, \mathscr{F}, \mu)$ and $M a \mathscr{T}_{c}$-closed submodule of $S$ such that both $S$ and $M$ have the countable concatenation property. Then $S=M \oplus M^{\perp}$.

Proof. It follows from Propositions 34 and 35 and Theorem 47.

Corollary 50. Let $(S,\langle\cdot, \cdot\rangle)$ be a $\mathscr{T}_{c}$-complete RIP module over $K$ with base $(\Omega, \mathscr{F}, \mu)$ such that $S$ has the countable concatenation property. Then for each $f \in S^{*}$ there exists a unique $y_{f} \in S$ such that $f(x)=\left\langle x, y_{f}\right\rangle$, for all $x \in S$, and $\|f\|=\left\|y_{f}\right\|$.

Proof. It follows from Proposition 35 and Theorem 48.

Remark 51. Based on Theorem 48, we can establish the spectral representation theorem for random self-adjoint operators on complete complex random inner product modules, which has been used to establish the Stone's representation theorem for a group of random unitary operators in [24].

\section{5. $\left(L^{p}(S)\right)^{\prime} \cong L^{q}\left(S^{*}\right)$}

In this section, let $(S,\|\cdot\|)$ be a given $\mathrm{RN}$ module over $K$ with base $(\Omega, \mathscr{F}, \mu)$ and $S^{*}$ its random conjugate space. Further, let $1 \leq p<+\infty$ and $1<q \leq+\infty$ be a pair of Hölder conjugate numbers.

Let $r$ be an extended nonnegative number with $1 \leq r \leq$ $+\infty$ and $\left(L^{r}(\mathscr{F}),|\cdot|_{r}\right)$ the Banach space of equivalence classes of $r$-integrable (when $r<+\infty$ ) or essentially bounded (when $r=+\infty)$ real $\mathscr{F}$-measurable functions on $(\Omega, \mathscr{F}, \mu)$ with the usual $L^{r}$-norm $|\cdot|_{r}$. Further, let $L^{r}(S)=\{x \in S \mid\|x\| \epsilon$ $\left.L^{r}(\mathscr{F})\right\}$ and let $\|\cdot\|_{r}: L^{r}(S) \rightarrow[0,+\infty)$ be defined by $\|x\|_{r}=|\|x\||_{r}$, for all $x \in L^{r}(S)$; then $\left(L^{r}(S),\|\cdot\|_{r}\right)$ is a normed space over $K$ and $\mathscr{T}_{\epsilon, \lambda}$-dense in $S$; compare [22, 25]. Similarly, one can understand the implication of $L^{r}\left(S^{*}\right)$.

Theorem 52 below is proved in [25], a more general result is proved in [6] with $L^{r}(\mathscr{F})$ replaced by a Köthe function space, but the two proofs both only give the key idea of them. Here, we give a detailed proof of Theorem 52. Since our aim is to look for the tool for the development of the theory of RN modules together with their random conjugate spaces, Theorem 52 is enough for the aim.

Theorem 52. $L^{q}\left(S^{*}\right) \cong\left(L^{p}(S)\right)^{\prime}$ under the canonical mapping $T$, where $\left(L^{p}(S)\right)^{\prime}$ denotes the classical conjugate space of $L^{p}(S)$ 
and for each $f \in L^{q}\left(S^{*}\right), T_{f}$ (denoting $\left.T(f)\right): L^{p}(S) \rightarrow K$ is defined by $T_{f}(x)=\int_{\Omega} f(x) d \mu$, for all $x \in L^{p}(S)$.

We will divide the proof of Theorem 52 into the following two Lemmas-Lemmas 53 and 54.

Lemma 53. $T$ is isometric.

Proof. $\left|T_{f}(x)\right|=\left|\int_{\Omega} f(x) d \mu\right| \leq \int_{\Omega}|f(x)| d \mu \leq \int_{\Omega}\|f\|\|x\|$ $d \mu \leq\|f\|_{q}\|x\|_{p}$, for all $x \in L^{p}(S)$, so $\left\|T_{f}\right\| \leq\|f\|_{q}$.

As for $\|f\|_{q} \leq\left\|T_{f}\right\|$, we can, without loss of generality, assume that $(\Omega, \mathscr{F}, \mu)$ is a probability space. Let $\left\{x_{n}, n \in N\right\}$ be a sequence in $S(1):=\{x \in S \mid\|x\| \leq 1\}$ such that $\left\{\left|f\left(x_{n}\right)\right|, n \in N\right\}$ converges a.s. to $\|f\|$ in a nondecreasing manner (such a sequence $\left\{x_{n}, n \in N\right\}$ does exist!).

When $p=1$ and $q=+\infty$, for any positive number $\epsilon$ let $A(\epsilon)=\left[\|f\|>\|f\|_{\infty}-\epsilon\right]$; then $\mu(A(\epsilon))>0$, and hence there exists some $n_{0} \in N$ such that $B(\epsilon):=\left[\left|f\left(x_{n_{0}}\right)\right|>\|f\|_{\infty}-\epsilon\right]$ has a positive measure. Let $x_{\epsilon}=1 / \mu(B(\epsilon)) \cdot I_{B(\epsilon)} \cdot \overline{\operatorname{sgn}\left(f\left(x_{n_{0}}\right)\right)} \cdot x_{n_{0}}$; then $\left\|x_{\epsilon}\right\|_{1} \leq 1$ and $\left|T_{f}\left(x_{\epsilon}\right)\right|>\|f\|_{\infty}-\epsilon$, which shows that $\left\|T_{f}\right\| \geq\|f\|_{\infty}$.

When $p>1$,

$$
\begin{aligned}
\int_{\Omega}\left|f\left(x_{n}\right)\right|^{q} d \mu & =\int_{\Omega}\left|f\left(x_{n}\right)\right|^{q-1}\left|f\left(x_{n}\right)\right| d \mu \\
& =\int_{\Omega}\left|f\left(x_{n}\right)\right|^{q-1} \cdot \overline{\operatorname{sgn}\left(f\left(x_{n}\right)\right)} \cdot f\left(x_{n}\right) d \mu \\
& =\int_{\Omega} f\left(\left|f\left(x_{n}\right)\right|^{q-1} \cdot \overline{\operatorname{sgn}\left(f\left(x_{n}\right)\right)} \cdot x_{n}\right) d \mu \\
& =T_{f}\left(\left|f\left(x_{n}\right)\right|^{q-1} \cdot \overline{\operatorname{sgn}\left(f\left(x_{n}\right)\right)} \cdot x_{n}\right) \\
& \leq\left\|T_{f}\right\|\left(\int_{\Omega}\left|f\left(x_{n}\right)\right|^{q} d \mu\right)^{1 / p},
\end{aligned}
$$

then $\left\|f\left(x_{n}\right)\right\|_{q} \leq\left\|T_{f}\right\|$, for all $n \in N$. By the Levy theorem we have that $\|f\|_{q} \leq\left\|T_{f}\right\|$.

Lemma 54. $T$ is surjective.

Proof. For any fixed $l \in\left(L^{p}(S)\right)^{\prime}$ and $x \in L^{\infty}(S)$, define the scalar measure $G_{x}: \mathscr{F} \rightarrow K$ and the vector measure $G$ : $\mathscr{F} \rightarrow\left(L^{\infty}(S)\right)^{\prime}$ as follows:

$$
\begin{aligned}
& G_{x}(E)=l\left(\widetilde{I}_{E} \cdot x\right), \text { for all } E \in \mathscr{F}, \\
& G(E)(y)=l\left(\widetilde{I}_{E} \cdot y\right), \text { for all } y \in L^{\infty}(S) \text { and } E \in \mathscr{F} .
\end{aligned}
$$

Since $|G(E)(y)|=\left|l\left(\widetilde{I}_{E} \cdot y\right)\right| \leq\|l\| \cdot\left\|\widetilde{I}_{E} \cdot y\right\|_{p} \leq\|l\| \cdot\|y\|_{\infty}$. $\left\|\widetilde{I}_{E}\right\|_{p}$, for all $E \in \mathscr{F}, y \in L^{\infty}(S)$, both $G$ and $G_{x}$ are countably additive. Now, for any finite partition $\left\{E_{1}, E_{2}, \ldots, E_{n}\right\}$ of $\Omega$ to $\mathscr{F}$ and finitely many points $x_{1}, x_{2}, \ldots, x_{n}$, in the closed unit ball of $L^{\infty}(S)$, we have $\left|\sum_{i=1}^{n} G\left(E_{i}\right)\left(x_{i}\right)\right|=\left|l\left(\sum_{i=1}^{n} \widetilde{I}_{E_{i}} \cdot x_{i}\right)\right| \leq$ $\|l\| \cdot\left|\sum_{i=1}^{n} \widetilde{I}_{E_{i}}\right|_{p}=\|l\|$. Similarly, we have that $\sum_{i=1}^{n}\left|G_{x}\left(E_{i}\right)\right|=$ $\sum_{i=1}^{n} \overline{\operatorname{sgn}\left(G_{x}\left(E_{i}\right)\right)} \cdot G_{x}\left(E_{i}\right)=\sum_{i=1}^{n} G\left(E_{i}\right)\left(\overline{\operatorname{sgn}\left(G_{x}\left(E_{i}\right)\right)} \cdot x\right) \leq$ $\|l\| \cdot\|x\|_{\infty}$. So $|G|(\Omega) \leq\|l\|$ and $\left|G_{x}\right|(\Omega) \leq\|l\| \cdot\|x\|_{\infty}$; namely, $G$ and $G_{x}$ are both of bounded variation and they are both absolutely continuous with respect to $\mu$.

By the classical Radon-Nikodým theorem there exists a unique $g(x) \in L^{1}(\mathscr{F}, K)$ for each $x \in L^{\infty}(S)$ such that $G_{x}(E)=\int_{E} g(x) d \mu$, for all $E \in \mathscr{F}$, and $\left|G_{x}\right|(E)=\int_{E}|g(x)| d \mu$, for all $E \in \mathscr{F}$, so we can obtain a mapping $g: L^{\infty}(S) \rightarrow$ $L^{1}(\mu, K)$ such that

(1) $g(\alpha x+\beta y)=\alpha g(x)+\beta g(y)$, for all $\alpha, \beta \in K$, and $x, y \in L^{\infty}(S)$;

(2) $g(\xi x)=\xi \cdot g(x)$ for each simple element $\xi$ in $L^{0}(\mathscr{F}, K), x \in L^{\infty}(S)$.

We can now assert that $g(\xi x)=\xi g(x)$, for all $\xi \epsilon$ $L^{\infty}(\mathscr{F}, K)$ and $x \in L^{\infty}(S)$. In fact, for any $\xi \in L^{\infty}(\mathscr{F}, K)$ there are always a sequence $\left\{\xi_{n}, n \in N\right\}$ of simple elements in $L^{0}(\mathscr{F}, K)$ such that $\left\{\left\|\xi_{n}-\xi\right\|_{\infty}, n \in N\right\}$ converges to 0 ; then $\left\|g(\xi x)-g\left(\xi_{n} x\right)\right\|_{1}=\left|G_{\left(\xi-\xi_{n}\right) x}\right|(\Omega) \leq\|l\| \cdot\left\|\xi-\xi_{n}\right\|_{\infty} \cdot\|x\|_{\infty} \rightarrow$ $0(n \rightarrow \infty)$. On the other hand, $\left\|\xi g(x)-\xi_{n} g(x)\right\|_{1} \leq$ $\left\|\xi-\xi_{n}\right\|_{\infty}\|g(x)\|_{1} \rightarrow 0(n \rightarrow \infty)$, so $g(\xi x)=L^{1}-$ $\lim _{n \rightarrow \infty} g\left(\xi_{n} x\right)=L^{1}-\lim _{n \rightarrow \infty}\left(\xi_{n} g(x)\right)=\xi g(x)$.

We prove that $\{|g(x)||x \in S(1)|\}$ is upward directed as follows: for any $x$ and $y \in S(1)$, let $E=[|g(x)| \leq|g(y)|]$; then $|g(x)| \vee|g(y)|=I_{E}|g(y)|+\left(1-I_{E}\right)|g(x)|=g\left(\overline{\operatorname{sgn}(g(y))} \cdot I_{E} \cdot y+\right.$ $\left.\overline{\operatorname{sgn}(g(x))} \cdot\left(1-I_{E}\right) \cdot x\right)=g(z)=|g(z)|$, where $z=\overline{\operatorname{sgn}(g(y))} \cdot I_{E}$. $y+\overline{\operatorname{sgn}(g(x))} \cdot\left(1-I_{E}\right) \cdot x \in S(1)$. Hence, there exists a sequence $\left\{x_{n}, n \in N\right\}$ in $S(1)$ such that $\left\{\left|g\left(x_{n}\right)\right|, n \in N\right\}$ converges a.s. to $\xi:=\vee\{|g(x)| \mid x \in S(1)\}$ in a nondecreasing manner. By the Levy theorem $\|\xi\|_{1}=\lim _{n \rightarrow \infty}\left\|g\left(x_{n}\right)\right\|_{1}=\lim _{n \rightarrow \infty}\left|G_{x_{n}}\right|(\Omega) \leq$ $|G|(\Omega) \leq\|l\|<+\infty$, so $\xi \in L^{1}(\mathscr{F}, K)$. Since for any positive number $\epsilon$ and $x \in L^{\infty}(S)$, it is clear that $|g((1 /(\|x\|+\epsilon)) x)| \leq$ $\xi$; namely, $|g(x)| \leq \xi(\|x\|+\epsilon)$, which implies that $|g(x)| \leq$ $\xi\|x\|$, for all $x \in L^{\infty}(S)$. By the Hahn-Banach theorem for a.s. bounded random linear functionals (see $[2,8])$ there is an a.s. bounded random linear functional $f \in S^{*}$ such that $\left.f\right|_{L^{\infty}(S)}=g$; further $f$ is unique since $L^{\infty}(S)$ is $\mathscr{T}_{\epsilon, \lambda}$-dense in $S$ and $\|f\| \leq \xi$; we also have that $\|f\| \in L^{1}(\mathscr{F}, K)$.

By the definition of $G_{x}, l(x)=G_{x}(\Omega)=\int_{\Omega} g(x) d \mu=$ $\int_{\Omega} f(x)$, for all $x \in L^{\infty}(S)$. We prove that $f \in L^{q}\left(S^{*}\right)$ as follows: let $E_{n}=[\|f\| \leq n]$ and $f_{n}=I_{E_{n}} \cdot f$; then $f_{n} \in L^{q}\left(S^{*}\right)$ and $\left\|f_{n}\right\|=I_{E_{n}}\|f\|$ (here, we can assume that $\mu$ is a probability measure). Since $\int_{E_{n}} f(x) d \mu=l\left(I_{E_{n}} \cdot x\right)$, for all $x \in L^{\infty}(S)$ and $n \in N$, then $\left.T_{f_{n}}\right|_{L^{\infty}(S)}=\left.l\right|_{I_{E} \cdot L^{\infty}(S)}$. From the fact that $L^{\infty}(S)$ is dense in $\left(L^{p}(S),\|\cdot\|_{p}\right),\left.T_{f_{n}}\right|_{L^{p}(S)}=l_{I_{E_{n}} \cdot L^{p}(S)}$, so $\left\|T_{f_{n}}\right\| \leq\|l\|$; letting $n \rightarrow+\infty$ yields that $\|f\|_{q} \leq \lim _{n \rightarrow \infty}\left\|f_{n}\right\|_{q}=$ $\lim _{n \rightarrow \infty}\left\|T_{f_{n}}\right\| \leq\|l\|<+\infty$; namely, $f \in L^{q}\left(S^{*}\right)$. Further, we also have that $l=T_{f}$ since they coincide on the dense subspace $L^{\infty}(S)$ of $L^{p}(S)$.

Remark 55. Let $(B,\|\cdot\|)$ be a normed space over $K$ and $L^{0}(\mathscr{F}, B)$ the $L^{0}(\mathscr{F}, K)$-module of equivalence classes of $B$ - 
valued $\mathscr{F}$-strongly measurable functions on $(\Omega, \mathscr{F}, \mu)$. Let $B^{\prime}$ be the classical conjugate space of $B$ and $L^{0}\left(\mathscr{F}, B^{\prime}, w^{*}\right)$ the $L^{0}(\mathscr{F}, K)$-module of $w^{*}$-equivalence classes of $B^{\prime}$-valued $w^{*}$ measurable functions on $(\Omega, \mathscr{F}, \mu)$. For any $x \in L^{0}(\mathscr{F}, B)$ with a representative $x^{0}$, the $L^{0}$-norm of $x$ is defined to be the equivalence class of $\left\|x^{0}\right\|$, still denoted by $\|x\|$; then $\left(L^{0}(\mathscr{F}, B),\|\cdot\|\right)$ is an RN module over $K$ with base $(\Omega, \mathscr{F}, \mu)$. For any $y \in L^{0}\left(\mathscr{F}, B^{\prime}, w^{*}\right)$ with a representative $y^{0}$, the $L^{0}$-norm of $y$ is defined to be the equivalence class of $\operatorname{esssup}\left\{\left|y^{0}(b)\right| \quad \mid \quad b \in B\right.$ and $\left.\|b\| \leq 1\right\}$ (namely, the essential supremum of $\left\{\left|y^{0}(b)\right| \quad \mid \quad b \in B\right.$ and $\|b\| \leq$ $1\})$; then $L^{0}\left(\mathscr{F}, B^{\prime}, w^{*}\right)$ is also an RN module over $K$ with base $(\Omega, \mathscr{F}, \mu)$. In [26] it is proved that $\left(L^{0}(\mathscr{F}, B)\right)^{*}=$ $L^{0}\left(\mathscr{F}, B^{\prime}, w^{*}\right)$, so if we take $S=L^{0}(\mathscr{F}, B)$ in Theorem 52 then $L^{p}(\mathscr{F}, B)^{\prime} \cong L^{q}\left(\mathscr{F}, B^{\prime}, w^{*}\right)$. Generally speaking, $\sigma$-finite measure spaces are enough for various kinds of problems in analysis, but some more general measure spaces are sometimes necessary; for example, strictly localizable measure spaces are considered in [6]. Even in [27] we introduced the notion of an RN module with base being an arbitrary measure space $(\Omega, \mathscr{F}, \mu)$ by defining it to be a projective limit of a family of RN modules with base $\left(A, A \cap \mathscr{F},\left.\mu\right|_{A \cap \mathscr{F}}\right)$, where $A \in \mathscr{F}$ satisfies $0<\mu(A)<+\infty$, and further proved that Theorem 52 remains true for any measure space, so Theorem 52 unifies all the representation theorems of the dual spaces of Lebesgue-Bochner function spaces. As said in $[6,25]$, it is more interesting that Theorem 52 establishes the key connection between random conjugate spaces and classical conjugate spaces, which has played a crucial role in the subsequent development of random conjugate spaces; compare $[22,28,29]$.

Remark 56. Since the Lebesgue-Bochner function space $L^{p}(\mathscr{F}, B)$ (or is written as $L^{p}(\mu, B)$ ) has the target space $B$, the simple functions in $L^{p}(\mathscr{F}, B)$ always play an active role in the study of the dual of $L^{p}(\mathscr{F}, B)$, whereas we do not have the counterparts of simple elements in $L^{p}(\mathscr{F}, B)$ for abstract spaces $L^{p}(S)$, so we are forced to replace simple elements in $L^{p}(\mathscr{F}, B)$ with elements in $L^{\infty}(S)$ in order to complete the proof of Theorem 52. In [26] we prove that a Banach space $B$ is reflexive if and only if $L^{0}(\mathscr{F}, B)$ is random reflexive; the original motivation of Theorem 52 is to establish the following characterization.

Theorem 57. Let $(S,\|\cdot\|)$ be a $\mathscr{T}_{\epsilon, \lambda}$-complete $R N$ module over $K$ with base $(\Omega, \mathscr{F}, \mu)$ and $p$ any given positive number such that $1<p<+\infty$. Then $S$ is random reflexive if and only if $L^{p}(S)$ is reflexive.

Proof. Let $J: S \rightarrow S^{* *}$ and $j: L^{p}(S) \rightarrow\left(L^{p}(S)\right)^{\prime \prime}$ be the corresponding canonical embedding mappings.

(1) Necessity. Since $1<p<+\infty$, its Hölder conjugate number $q$ satisfies $1<q<+\infty$; then $\left(L^{p}(S)\right)^{\prime \prime}=\left(L^{q}\left(S^{*}\right)\right)^{\prime}=$ $L^{p}\left(S^{* *}\right)=L^{p}(S)$.

(2) Sufficiency. Let $f^{* *}$ be any given element in $S^{* *}$ and $E_{n}=$ $\left[n-1 \leq\left\|f^{* *}\right\|<n\right]$ for any $n \in N$. We can, without loss of generality, assume that $\mu$ is a probability measure; then $\sum_{n=1}^{\infty} \mu\left(E_{n}\right)=1$. Since $I_{E_{n}} f^{* *} \in L^{p}\left(S^{* *}\right)=\left(L^{p}(S)\right)^{\prime \prime}$, there exists $x_{n} \in L^{p}(S)$ such that $j\left(x_{n}\right)=I_{E_{n}} f^{* *}$; namely, for each $f^{*} \in L^{q}\left(S^{*}\right)$, we have that $\int_{\Omega} f^{*}\left(x_{n}\right) d \mu=j\left(x_{n}\right)\left(f^{*}\right)=$ $\int_{\Omega} I_{E_{n}} f^{* *}\left(f^{*}\right) d \mu$. By replacing $f^{*}$ with $I_{E} f^{*}$ we can obtain that $\int_{E} f^{*}\left(x_{n}\right) d \mu=\int_{E} I_{E_{n}} f^{* *}\left(f^{*}\right) d \mu$, for all $E \in \mathscr{F}$ and $f^{*} \in L^{q}\left(S^{*}\right)$, which implies that $f^{*}\left(x_{n}\right)=I_{E_{n}} f^{* *}\left(f^{*}\right)$, for all $f^{*} \in L^{q}\left(S^{*}\right)$. Since $L^{q}\left(S^{*}\right)$ is $\mathscr{T}_{\epsilon, \lambda}$-dense in $S^{*}, J\left(x_{n}\right)=I_{E_{n}} f^{* *}$, for all $n \in N$.

Let $y_{n}=\sum_{k=1}^{n} I_{E_{k}} \cdot x_{k}$, for all $n \in N$; then $\left\{y_{n}, n \in N\right\}$ is $\mathscr{T}_{\epsilon, \lambda}$-Cauchy in $S$ and hence convergent to some $y \in S$, which shows that $J(y)=\lim _{n \rightarrow \infty} J\left(y_{n}\right)=\lim _{n \rightarrow \infty} \sum_{k=1}^{n} I_{E_{k}} \cdot f^{* *}=$ $\left(\sum_{n=1}^{\infty} I_{E_{n}}\right) \cdot f^{* *}=f^{* *}$; namely, $J$ is surjective.

Remark 58. Concerning Theorem 57, a similar and more general result was given in [6] where $L^{p}(\mathscr{F})$ is replaced by a reflexive Köthe function space.

\section{Acknowledgment}

The author would like to thank Professor Quanhua Xu for kindly providing the reference [6] in February 2012, which makes us know, for the first time, the excellent work of Haydon, Levy, and Raynaud.

\section{References}

[1] B. Schweizer and A. Sklar, Probabilistic Metric Spaces, Elsevier, New York, NY, USA, 1983, Reissued by Dover Publications, New York, NY, USA, 2005.

[2] T. X. Guo, The theory of probabilistic metric spaces with applications to random functional analysis [M.S. thesis], Xian Jiaotong University, Xi'an, China, 1989.

[3] T. X. Guo, "Some basic theories of random normed linear spaces and random inner product spaces," Acta Analysis Functionalis Applicata, vol. 1, no. 2, pp. 160-184, 1999.

[4] T. X. Guo, "The relation of Banach-Alaoglu theorem and Banach-Bourbaki-Kakutani-Šmulian theorem in complete random normed modules to stratification structure," Science in China. Series A, vol. 51, no. 9, pp. 1651-1663, 2008.

[5] T. X. Guo, Random metric theory and its applications [Ph.D. thesis], Xi'an Jiaotong University, Xian, China, 1992.

[6] R. Haydon, M. Levy, and Y. Raynaud, Randomly Normed Spaces, Hermann, Paris, France, 1991.

[7] D. Filipović, M. Kupper, and N. Vogelpoth, "Separation and duality in locally $L^{0}$-convex modules," Journal of Functional Analysis, vol. 256, no. 12, pp. 3996-4029, 2009.

[8] T. X. Guo, "Relations between some basic results derived from two kinds of topologies for a random locally convex module," Journal of Functional Analysis, vol. 258, no. 9, pp. 3024-3047, 2010.

[9] T. X. Guo and Y. J. Yang, "Ekeland's variational principle for an $\bar{L}^{0}$-valued function on a complete random metric space," Journal of Mathematical Analysis and Applications, vol. 389, no. 1, pp. 1-14, 2012.

[10] S. E. Zhao and T. X. Guo, "The random subreflexivity of complete random normed modules," International Journal of Mathematics, vol. 23, no. 3, Article ID 1250047, 14 pages, 2012. 
[11] T. X. Guo, "Recent progress in random metric theory and its applications to conditional risk measures," Science China. Series A, vol. 54, no. 4, pp. 633-660, 2011.

[12] T. X. Guo, S. E. Zhao, and X. L. Zeng, "On random convex analysis-the analytic foundation of the module approach to conditional risk measures," http://arxiv.org/abs/1210.1848.

[13] N. Dunford and J. T. Schwartz, Linear Operators(I), Interscience, New York, NY, USA, 1957.

[14] T. X. Guo, "Module homomorphisms on random normed modules," Northeastern Mathematical Journal (China), vol. 12, no. 1, pp. 102-114, 1996.

[15] J. P. LaSalle, "Pseudo-normed linear spaces," Duke Mathematical Journal, vol. 8, pp. 131-135, 1941.

[16] T. X. Guo, "Metrics, probabilistic metrics and random metrics," Systems Science and Mathematical Sciences (China), vol. 8, no. 2, pp. 182-186, 1995.

[17] Z. Y. You and L. H. Zhu, "The isometric metrization of probabilistic metric spaces," Scientia Sinica, Series A, vol. 1, pp. 19-24, 1989.

[18] K. Yosida, Functional Analysis, vol. 123, Beijing World Publishing, Beijing, China, 6th edition, 1999.

[19] T. X. Guo, "Extension theorems of continuous random linear operators on random domains," Journal of Mathematical Analysis and Applications, vol. 193, no. 1, pp. 15-27, 1995.

[20] T. X. Guo and Z. Y. You, "The Riesz representation theorem for random inner product modules and its applications," Chinese Annals of Mathematics. Series A, vol. 17, no. 3, pp. 361-364, 1996.

[21] Z. Y. You and T. X. Guo, "Pointwise best approximation in the space of strongly measurable functions with applications to best approximation in $L^{p}(\mu, X)$," Journal of Approximation Theory, vol. 78, no. 3, pp. 314-320, 1994.

[22] T. X. Guo and S. B. Li, "The James theorem in complete random normed modules," Journal of Mathematical Analysis and Applications, vol. 308, no. 1, pp. 257-265, 2005.

[23] T. X. Guo and Z. Y. You, "A note on pointwise best approximation," Journal of Approximation Theory, vol. 93, no. 2, pp. 344$347,1998$.

[24] T. X. Guo and X. Zhang, "Stone's representation theorem of a group of random unitary operators on complete complex random inner product modules," Scientia Sinica, Series A, vol. 42, pp. 181-202, 2012.

[25] T. X. Guo, "A characterization for a complete random normed module to be random reflexive," Journal of Xiamen University. Natural Science, vol. 36, no. 4, pp. 167-170, 1997.

[26] T. X. Guo, "Radon-Nikodým property of conjugate Banach spaces and $w^{*}$-equivalence theorems of $w^{*}$-measurable functions," Science in China. Series A., vol. 39, no. 10, pp. 1034-1041, 1996.

[27] T. X. Guo, "Representation theorems of the dual of LebesgueBochner function spaces," Science in China. Series A, vol. 43, no. 3, pp. 234-243, 2000.

[28] T. X. Guo, H. Xiao, and X. Chen, "A basic strict separation theorem in random locally convex modules," Nonlinear Analysis: Theory, Methods \& Applications A, vol. 71, no. 9, pp. 3794-3804, 2009.

[29] M. Z. Wu, "The Bishop-Phelps theorem in complete random normed modules endowed with the $(\epsilon, \lambda)$-topology," Journal of Mathematical Analysis and Applications, vol. 391, no. 2, pp. 648652, 2012. 


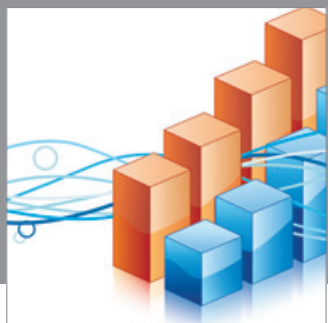

Advances in

Operations Research

mansans

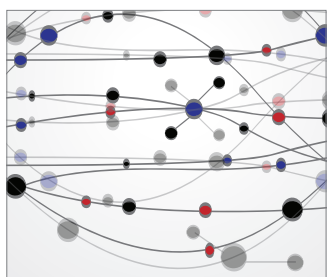

The Scientific World Journal
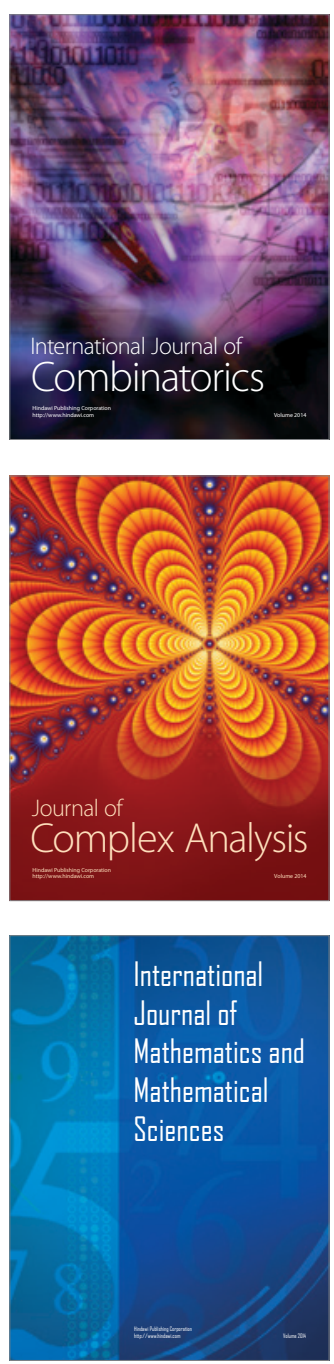
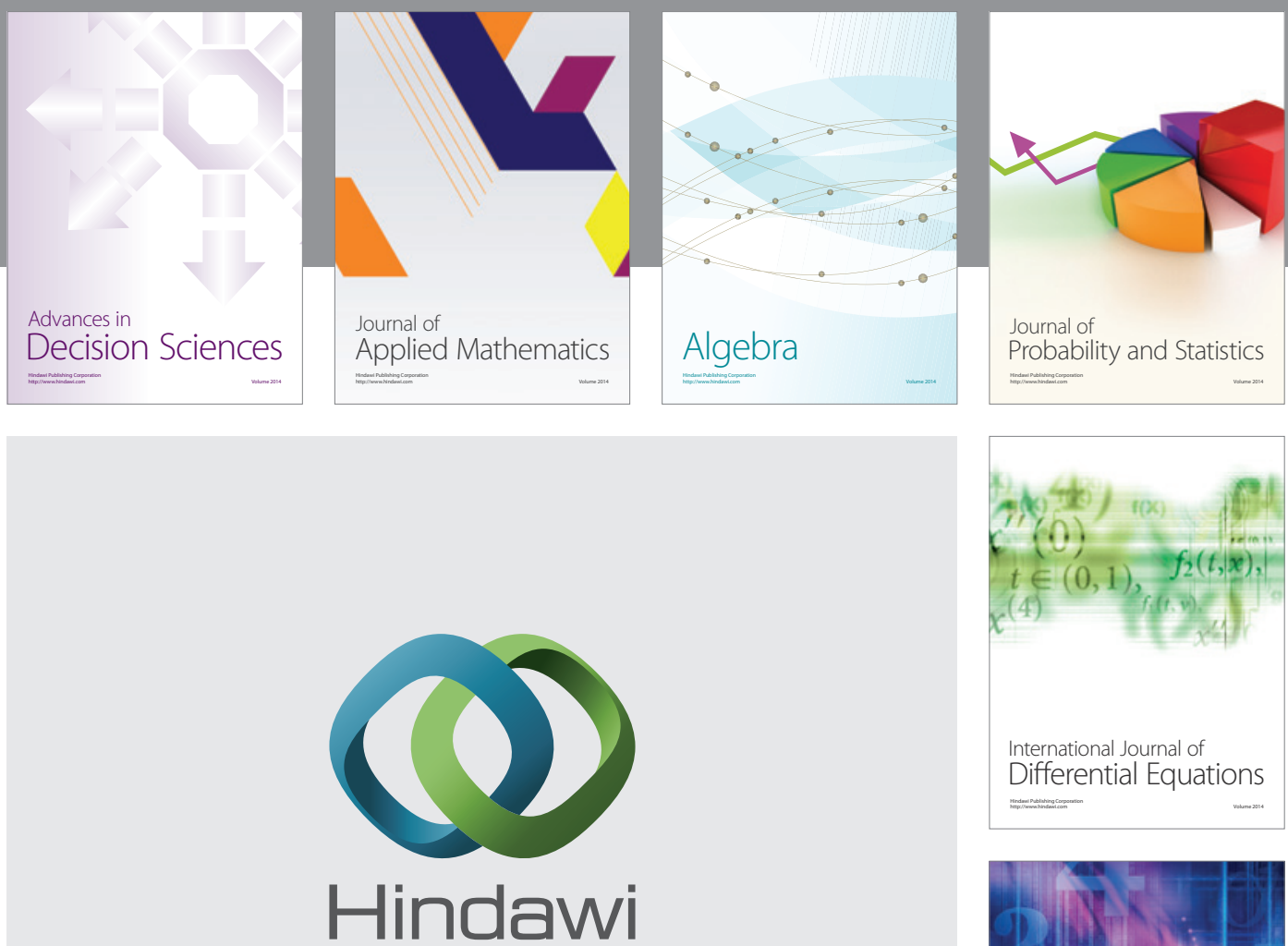

Submit your manuscripts at http://www.hindawi.com
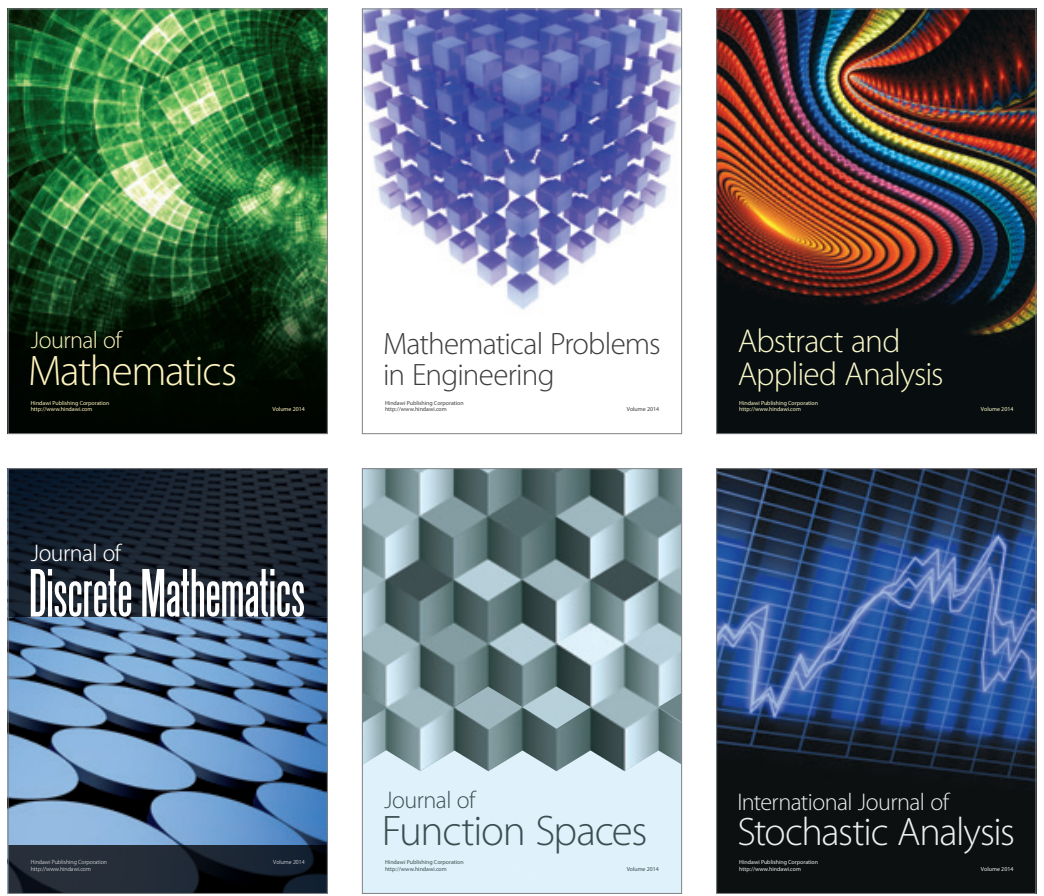

Journal of

Function Spaces

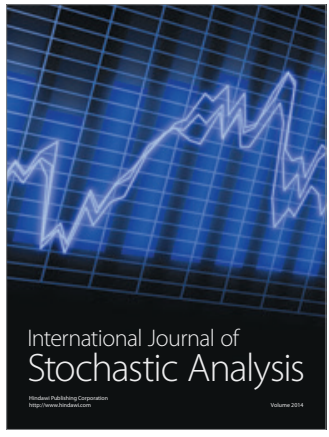

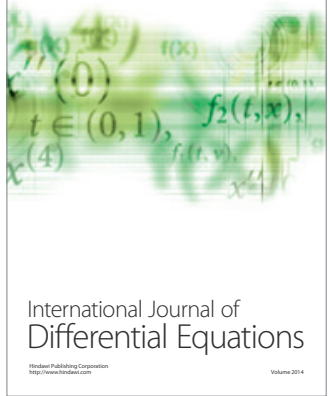
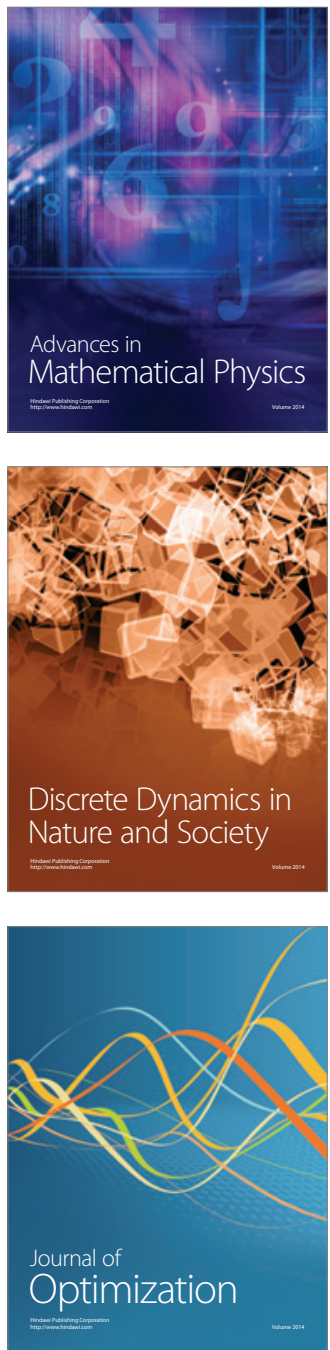